OPEN ACCESS

Edited by:

Hong Zhang,

Shanghai University of Traditional

Chinese Medicine, China

Reviewed by:

Jia-bo Wang,

Fifth Medical Center of the PLA

General Hospital, China

Haolong Liu,

Peking University Health Science

Centre, China

Lingyun Zhong,

Jiangxi University of Traditional

Chinese Medicine, China

*Correspondence:

Qin-wan Huang

huangqinwan@cdutcm.edu.cn

${ }^{\dagger}$ These authors share first authorship

Specialty section: This article was submitted to

Ethnopharmacology,

a section of the journal

Frontiers in Pharmacology

Received: 23 January 2020 Accepted: 13 May 2020

Published: 27 May 2020

Citation:

Gan Q-x, Wang J, Hu J, Lou G-h, Xiong $\mathrm{H}-\mathrm{j}$, Peng $\mathrm{C}-\mathrm{y}$ and Huang $\mathrm{Q}-\mathrm{W}$

(2020) Modulation of Apoptosis by

Plant Polysaccharides for Exerting

Anti-Cancer Effects: A Review.

Front. Pharmacol. 11:792.

doi: 10.3389/fphar.2020.00792

\section{Modulation of Apoptosis by Plant Polysaccharides for Exerting Anti- Cancer Effects: A Review}

\author{
Qing-xia Gan ${ }^{\dagger}$, Jin Wang ${ }^{\dagger}$, Ju Hu, Guan-hua Lou, Hai-jun Xiong, Cheng-yi Peng \\ and Qin-wan Huang*
}

College of Pharmacy, Chengdu University of Traditional Chinese Medicine, Chengdu, China

Cancer has become a significant public health problem with high disease burden and mortality. At present, radiotherapy and chemotherapy are the main means of treating cancer, but they have shown serious safety problems. The severity of this problem has caused further attention and research on effective and safe cancer treatment methods. Polysaccharides are natural products with anti-cancer activity that are widely present in a lot of plants, and many studies have found that inducing apoptosis of cancer cells is one of their important mechanisms. Therefore, this article reviews the various ways in which plant polysaccharides promote apoptosis of cancer cells. The major apoptotic pathways involved include the mitochondrial pathway, the death receptor pathway, and their upstream signal transduction such as MAPK pathway, PI3K/AKT pathway, and NF-KB pathway. Moreover, the paper has also been focused on the absorption and toxicity of plant polysaccharides with reference to extant literature, making the research more scientific and comprehensive. It is hoped that this review could provide some directions for the future development of plant polysaccharides as anticancer drugs in pharmacological experiments and clinical researches.

Keywords: plant polysaccharides, apoptosis, cancer, pathway, natural products

\section{INTRODUCTION}

Cancer is one of the most life-threatening diseases in the world (Zarei et al., 2016). In 2018, it has caused more than 9.6 million deaths as reported by the World Health Organization (WHO). The number of cancer patients continues to grow globally, despite the ongoing development of modern medical methods for preventing disease (López-Gómez et al., 2013; Surbone and Halpern, 2016; Srivastava et al., 2019). It is well known that the treatment of cancer is expensive and timeconsuming. The result is that cancer places a huge burden on individuals, families, communities, and health systems, and causes tremendous harm in physical, emotional, and financial of patients (Veenstra et al., 2014; Li Y. et al., 2018; Saad et al., 2019).

The occurrence of cancer is mainly caused by a series of changes in the genome and epigenome (Shen and Laird, 2013). These changes cause the cells to continuously proliferate and escape apoptosis, thereby disrupting the homeostasis of the tissue (Khan et al., 2016). It has been found that promoting cancer cell apoptosis is one of the effective methods to treat cancer (Mortezaee et al., 2019b). That is also the main mechanism of chemo(radio)therapy which is the most common 
cancer treatment (Agool et al., 2011; Deng et al., 2012; Mortezaee et al., 2019a). However, most of these drugs will cause normal cells apoptosis and lead to serious physical damage (Cheki et al., 2018; Zhang H. et al., 2018), such as myelosuppression, cardiotoxicity, hepatotoxicity, nephrotoxicity, and gastrointestinal toxicity (Liu et al., 2018; Oun et al., 2018; Zhang Q. et al., 2018). Finding safe and effective treatments has been a long-term goal of improving cancer.

Natural products isolated from plants are gradually recognized for their high efficiency and safety (Bishayee and Sethi, 2016). In cancer treatment, plant extracts have been widely used (Sun et al., 2019; Zabaiou et al., 2019). Polysaccharide is one of the active ingredients in a lot of plants, and shows low toxicity and high efficiency in the treatment of cancer (Jiao et al., 2016; Khan et al., 2019). Such as plant polysaccharides from genus Astragalus, Ginseng, Schisandra, and many others have been shown to be selective for the cytotoxicity of tumor cells, so they can kill cancer cells without the usually associated side effects. And the detailed molecular mechanism mainly involves the inhibition of cancer cell proliferation through promoting apoptosis. In recent years, studies have found that a variety of plant polysaccharides can regulate cancer cells apoptosis in vivo and in vitro. However, no systematic studies have evaluated the ability of plant polysaccharides to induce apoptosis. Therefore, this article reviews plant polysaccharides by inducing cancer cells apoptosis via multiple pathways and multiple targets to treat cancer, as shown in Figures 1-3 and Table 1, hoping to provide a reference for the treatment of cancer by plant polysaccharides in subsequent studies.

\section{CANCER AND APOPTOSIS}

Globally, cancer has been a serious burden on society, and the incidence of cancer is increasing (Zhang H. et al., 2018). In majority cancers, such as lung cancer, breast cancer, liver cancer, stomach cancer, laryngeal cancer, and prostate cancer, genetic mutations are common features (Rosell and Karachaliou, 2015).

\footnotetext{
Abbreviations: AKR1C2, aldo-keto reductase family 1 member C2; AKT, protein kinase b; AP-1, Activator Protein-1; Bad, Bcl-2-antagonist of cell death; Bak, BCL2 antagonist or killer; Bax, BCL-2-associated X protein; Bcl-2, B cell lymphoma 2; CDC, cell division cycle; Cdk, cyclin dependent kinases; COX- 2, prostaglandinendoperoxide synthase 2; CREB, the Cyclic AMP response element binding protein; ER $\alpha$, estrogen receptor alpha; FN, fibronectin; GSK-3 $\beta$, glycogen synthase kinase $3 \beta$; IGF-IR, insulin-like growth factor-I receptor; IKK, inhibitor

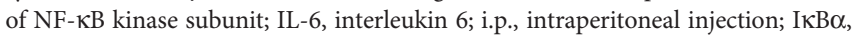
NF- $\kappa \mathrm{B}$ inhibitor alpha; JAK, Janus kinase; MAPK, mitogen activated protein kinase; Mcl-1, Myeloid cell leukemia-1; MMP, mitochondrial membrane potential; MMP-9, matrix metalloproteinase-9; MOMP, mitochondrial outer membrane permeabilization; MyD88, myeloid differentiation primary response gene 88 ; NF- $\kappa \mathrm{B}$, nuclear factor kappa B; Noxa, Apaf-1,apoptotic protease activating factor 1; PARP, poly (ADP-ribose) polymerase; PI3K, phosphoinositol-3-kinase; Puma, p53-upregulated modulator of apoptosis; PTTG1, pituitary tumor transforming gene 1; p-, phosphorylation; P38, p38 MAP kinase; ROS, reactive oxygen species; STAT, signal transducer and activator of transcription; TGF, transforming growth factor; TIMP, tissue inhibitors of metalloproteinase; TLR4, toll-like receptor 4; TNF, tumor necrosis factor; TNFR1, tumor necrosis factor receptor 1; TRAIL, TNF-related apoptosis-inducing ligand; VEGF, vascular endothelial growth factor.
}

One of the results of these genetic mutations is the inhibition of apoptosis. Cancer cells escape normal apoptosis, continue to proliferate, interfere with normal organs or tissues, and cause body damage or even death. Therefore, inducing apoptosis has always been one of the exact ways to inhibit cancer. The initiation of apoptosis is the opening or closing of a series of control switches after the corresponding signal stimulates the cell (Milisav et al., 2017). Different signal transduction triggers apoptosis in different ways (Zhang Q. et al., 2019). However, most of the apoptotic pathways eventually work by affecting a group of cysteine proteases called caspases which are revealed as the main executors of the apoptotic pathway due to their role in cleaving the major cellular substrate (Yaacoub et al., 2016). Caspase related to apoptosis is divided into two types, one is the initiator Caspases such as Caspase-2, Caspase-8, Caspase-9, and the other is the effector Caspases including Caspase-3, Caspase-6, Caspase-7 (Kopeina et al., 2018; Sadeghi et al., 2019). The initial Caspase is cleaved and activated under the action of foreign protein signals. The activated initiator cleaves and activates the effector, and finally lyses the cell substrate to cause apoptosis (Ludwig-Galezowska et al., 2011; Van Opdenbosch and Lamkanfi, 2019). Extrinsic death receptor pathway and mitochondrial pathway are two clear pathways that affect apoptosis. They can directly cause Caspase activation to exert a pro-apoptosis role through signaling of $\mathrm{Bcl}-2$ family proteins such as Bcl-2, Bax, Bad, and TNF families such as TNFR1, Fas, and TRAIL, respectively (Ludwig-Galezowska et al., 2011; Kaufmann et al., 2012). While most other pathways can indirectly affect Caspase proteins, such as MAPK, PI3K/AKT, and NF- $\kappa \mathrm{B}$ pathways can regulate apoptosis-related genes and ultimately stimulate mitochondria and death receptors to activate Caspase (Panka et al., 2006; Rohlenova et al., 2016).

\section{APOPTOSIS AND PLANT POLYSACCHARIDES}

Over the past decades, more than a hundred plant polysaccharides have been discovered, and in vitro and in vivo studies have shown that most of them have good anticancer activity in a variety of cancers. Lung cancer mice were treated with Scleromitrion diffusum (Willd.) R.J.Wang (syn. Hedyotis diffusa Willd.) polysaccharide (SDP) which consists of glucose, galactose, mannose ratio of 2.0: 1.0: 1.0. High-dose SDP exhibited tumor inhibition rates comparable to cisplatinpositive drugs (Lin et al., 2019). After treatment with Citrus $\times$ aurantiifolia (Christm.) Swingle polysaccharide (CAs) in transplanted $\mathrm{H} 22$ cells in mice, the tumor suppression rate was as high as $58.85 \%$ (Zhao Y. et al., 2017). Moreover, Polygonatum sibiricum Redouté polysaccharide had obvious anti-tumor effect on H22 tumor bearing mice (Duan et al., 2014). Similarly, Saccharina japonica (J.E. Areschoug) C.E. Lane, C. Mayes, Druehl \& G.W. Saunders (syn. Laminaria japonica) polysaccharide improved immunomodulatory activity and reduced tumor weight in H22-bearing mice. Simultaneously, the tumor suppression rate can reach 59.67\% (Zhu et al., 


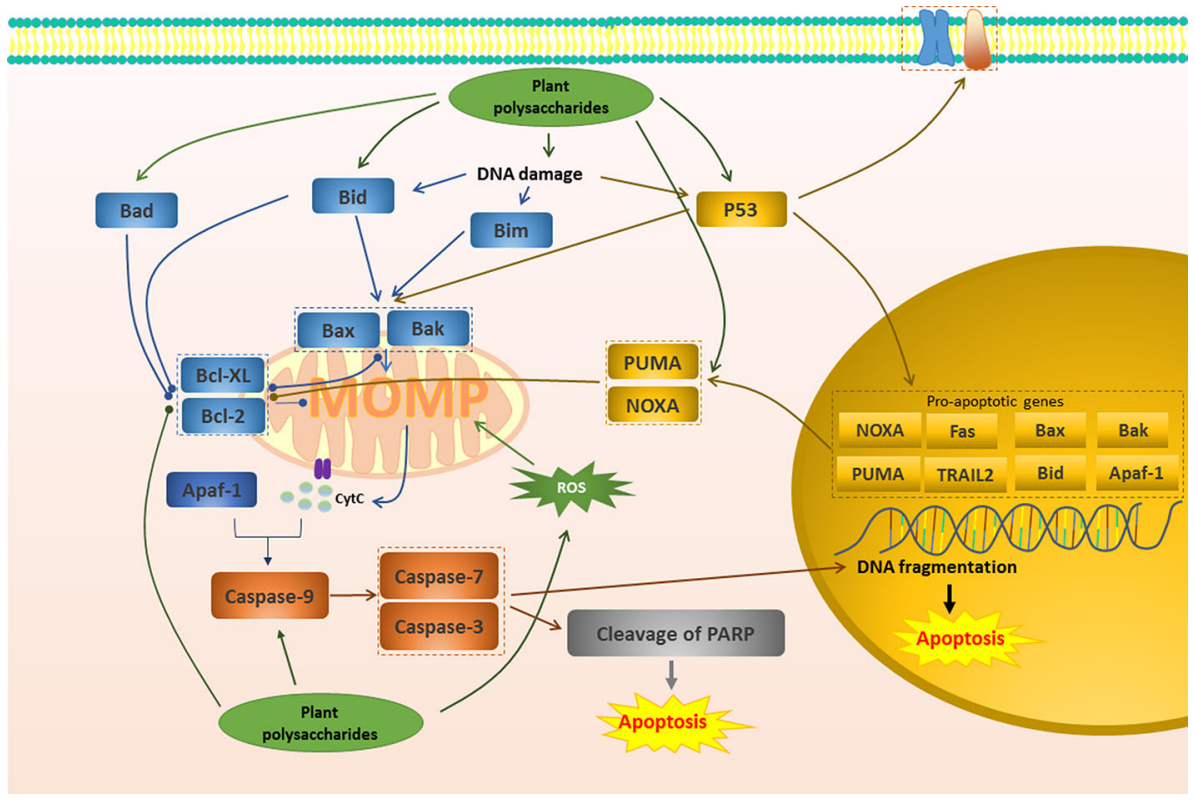

FIGURE 1 | Mitochondrial apoptotic pathway in cancer induced by plant polysaccharides. with different colors indicate inhibition/reduction, $\longrightarrow$ and $\longrightarrow$ with different colors indicate increase/promotion.

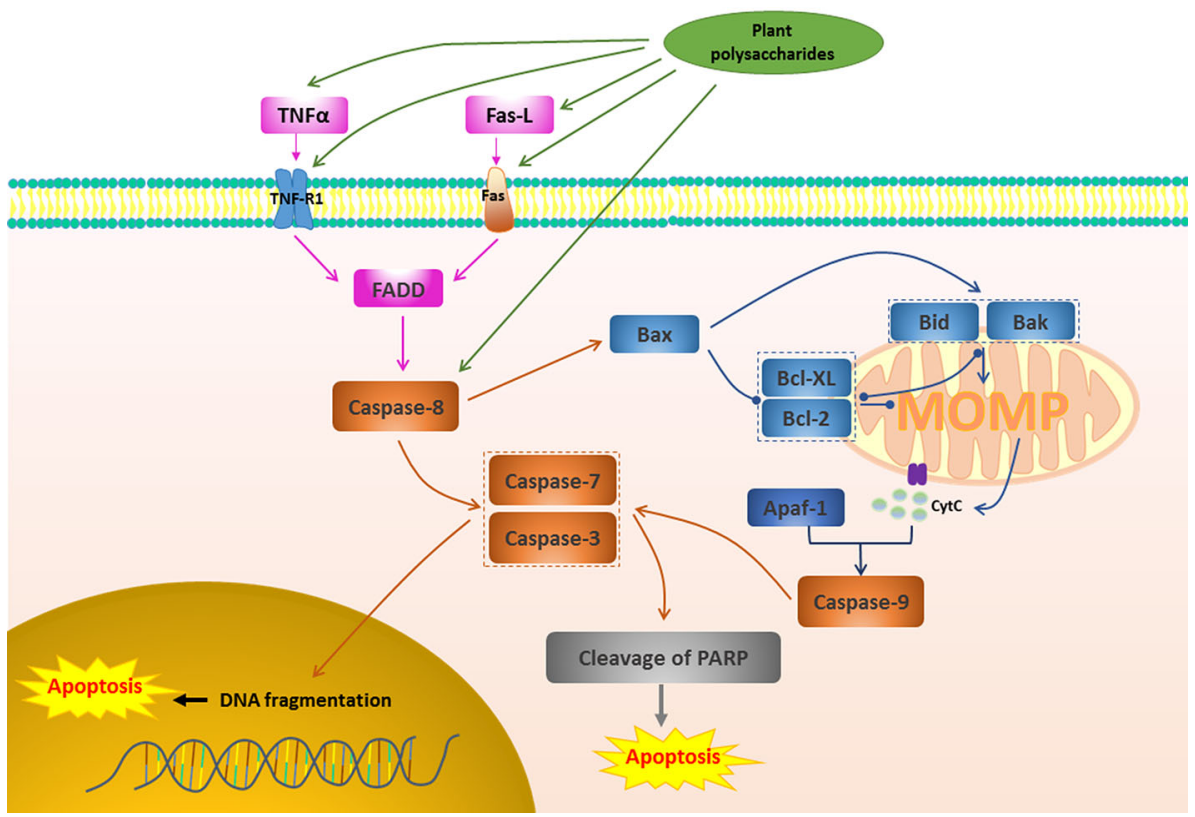

FIGURE 2 | Death receptor apoptotic pathway in cancer induced by plant polysaccharides. $\longrightarrow$ with different colors indicate inhibition/reduction, $\longrightarrow$ and $\longrightarrow$ with different colors indicate increase/promotion. 


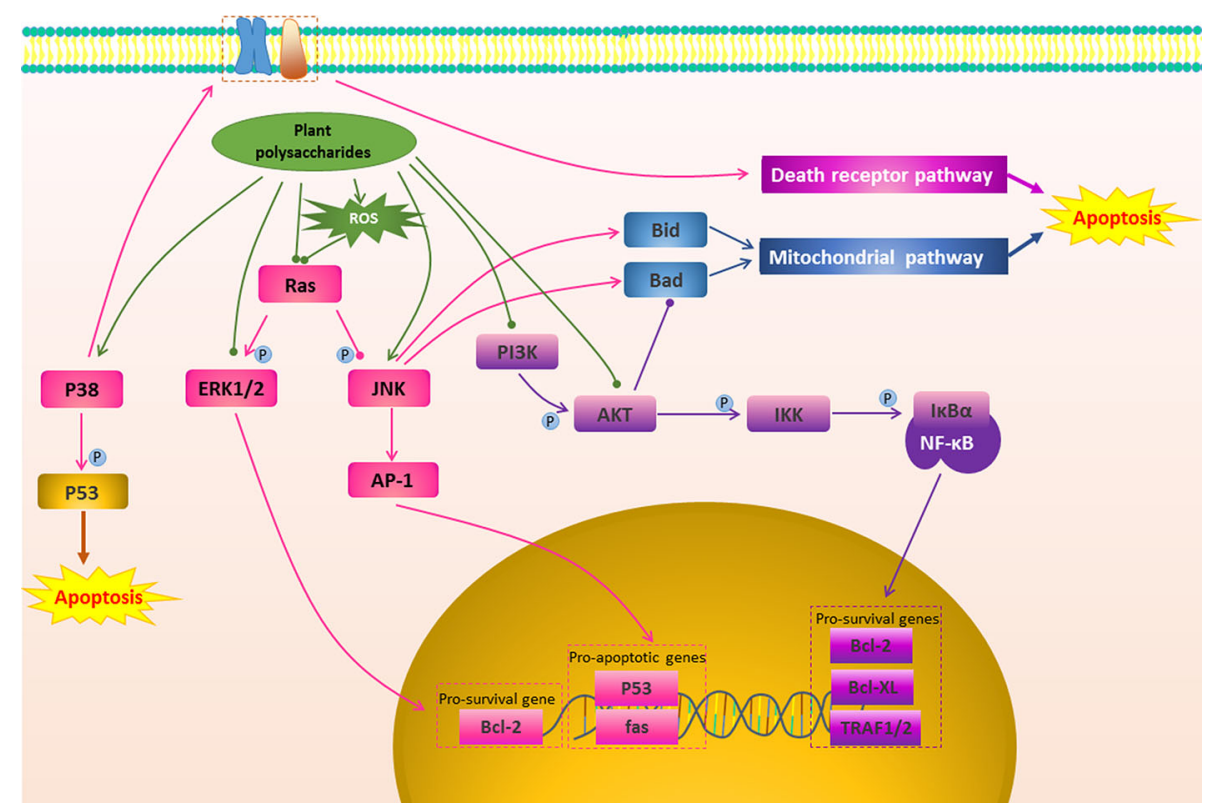

FIGURE 3 | The regulation of other apoptosis signal conduction in cancer by plant polysaccharides with different colors indicate inhibition/reduction, $\longrightarrow$ and $\longrightarrow$ with different colors indicate increase/promotion.

2016). The effect of Achyranthes bidentata Blume polysaccharide (ABPS) on tumor growth depended on its dose. At the doses of $100 \mathrm{mg} / \mathrm{kg}$ and $50 \mathrm{mg} / \mathrm{kg}$, ABPS inhibited the growth of mouse Lewis lung cancer by $5.36 \%$ and $40.06 \%$, respectively. It was further found that the anti-cancer activity of ABPS was mediated by inducing cell cycle arrest, and that high-dose ABPS stimulated tumor growth was related to NK cell dysfunction, upregulation of IL-6 and TNF- $\alpha$ (Jin et al., 2007).

Clinically, plant polysaccharides also show good curative effects, and are often combined with radiotherapy and chemotherapy. As a result, the toxic and side effects of radiotherapy and chemotherapy can be reduced, while improving the efficacy. Ginkgo biloba L. exopolysaccharide (GBEP) capsules were administered orally to 30 patients with gastric cancer. Compared with before treatment, GBEP could reduce the tumor area by $73.4 \%$. The changes of cell ultrastructure suggested that GBEP could induce tumor cell apoptosis and differentiation in patients with gastric cancer (Xu et al., 2003). In clinical trials, Ginseng polysaccharide injection was used in combination with chemotherapy to treat advanced malignancies such as lung, stomach and bowel cancer. The results showed that it could reduce the toxic and side effects of chemotherapy on patients, and improve the quality of life of patients, thereby enhancing the compliance of patient with chemotherapy. At the same time, Ginseng polysaccharide injection can improve the cellular immune function of patients and enhance the anti-cancer effect (Liu, 2008; Xu, 2015). In a phase II double-blind randomized placebo-controlled trial conducted by Astragalus polysaccharides (PG2), advanced head and neck squamous cell carcinoma (HNSCC) was treated with
PG2 and chemoradiation. PG2 was found that have the ability to improve the quality of life and adverse reactions that may be related to radiotherapy (Hsieh et al., 2020). Clinical studies have confirmed that Astragalus polysaccharides (APS) combined with I125, cisplatin, gemcitabine and platinum, respectively, all can treat non-small cell lung cancer, improve the immune function of patient, reduce the toxicity and side effects of chemo(radio) therapy on patients, and improve the overall quality of life (Qin et al., 2009; Guo et al., 2012; Sun et al., 2014).

On the whole, plant polysaccharides have good effects on the treatment of cancer, and they have excellent clinical value as adjuvant drugs for tumor chemo(radio)therapy. Understanding the therapeutic mechanism of drugs is conducive to a more scientific understanding and development of drugs. Studies have found that polysaccharides can achieve anticancer effects through a variety of mechanisms, such as preventing cancer cell metastasis, enhancing immune activity, and inhibiting division, while the main mechanism is inducing apoptosis of cancer cells (Zong et al., 2012; Khan et al., 2019). Therefore, we will summarize and analyze the specific mechanism of inhibiting cancer by inducing apoptosis of cancer cells by plant polysaccharides.

\section{MODULATION OF APOPTOSIS PATHWAY BY PLANT POLYSACCHARIDES IN CANCER}

\section{Mitochondrial Pathway}

The mitochondrial pathway begins with the apoptosis-regulating protein family represented by the Bcl-2 family (Wang et al., 


\begin{tabular}{|c|c|c|c|c|c|c|c|}
\hline Drugs & $\begin{array}{l}\text { Monosaccharide } \\
\text { composition/structure }\end{array}$ & Model & Dose and treat time & $\begin{array}{l}\text { Activity and type of } \\
\text { cancer }\end{array}$ & Apoptotic pathway & Involved mechanism & Ref \\
\hline $\begin{array}{l}\text { Andrographis } \\
\text { paniculata (Burm.f.) } \\
\text { Nees polysaccharide } \\
\text { (APWP) }\end{array}$ & Galatose, arabinose, rhamnose $(6: 3: 1)$ & HepG2 cells. & $\begin{array}{l}0-1,000 \mu \mathrm{g} / \mathrm{ml}, 24 \\
48, \text { and } 72 \mathrm{~h}\end{array}$ & $\begin{array}{l}\text { AR: } 65.1 \% \\
\text { CV: } 34.7 \% \\
\text { Hepatocellular } \\
\text { carcinoma }\end{array}$ & Mitochondrial Pathway & 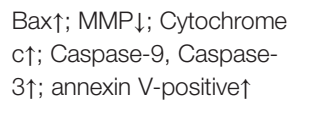 & (Zou et al., 2015) \\
\hline $\begin{array}{l}\text { Asparagus officinalis } L \text {. } \\
\text { polysaccharide (AP) }\end{array}$ & & MSC-2cells & $\begin{array}{l}0.25 \text {, and } 0.5 \mathrm{mg} / \mathrm{ml} \text {, } \\
72 \mathrm{~h}\end{array}$ & $\begin{array}{l}\text { AR: } 12.1 \% \\
\text { CV: About } 50 \% \\
\text { Colon cancer }\end{array}$ & Mitochondrial Pathway & 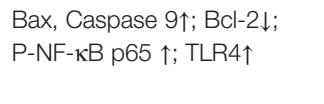 & $\begin{array}{l}\text { (Zhang W. et al., } \\
\text { 2018) }\end{array}$ \\
\hline $\begin{array}{l}\text { Artemisia capillaris } \\
\text { Thunb polysaccharide } \\
\text { (WACP) }\end{array}$ & Arabinose, galactose (4:2) & CNE-2 cells & $\begin{array}{l}0-200 \mu \mathrm{g} / \mathrm{ml}, 24,48 \\
72 \mathrm{~h}\end{array}$ & $\begin{array}{l}\text { AR: } 47.65 \% \\
\text { Nasopharyngeal } \\
\text { carcinoma }\end{array}$ & Mitochondrial Pathway & 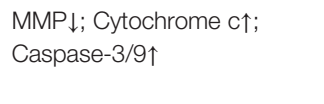 & (Feng et al., 2013) \\
\hline $\begin{array}{l}\text { Astragalus } \\
\text { mongholicus Bunge } \\
\text { (Astragali Radix) } \\
\text { Polysaccharides }\end{array}$ & $\begin{array}{l}\text { Rhamnose, arabinose, glucose, galactose, } \\
\text { Glucuronic acid (0.03:1.00:0.27:0.36:0.30) }\end{array}$ & SW620 cells & $0.1-1.0 \mathrm{~g} / \mathrm{L}, 48 \mathrm{~h}$ & $\begin{array}{l}\text { AR: } 37.63 \% \\
\text { Colon cancer }\end{array}$ & Mitochondrial Pathway & $\begin{array}{l}\text { Bax/Bcl-2 } \uparrow \text {; Caspase-3, } \\
\text { Caspase-9, Cytochrome c } \uparrow\end{array}$ & (Yan et al., 2017) \\
\hline $\begin{array}{l}\text { Astragalus } \\
\text { mongholicus Bunge } \\
\text { polysaccharide (APS) }\end{array}$ & & $\begin{array}{l}\text { Mice injected with } \\
\text { MDA-MB-231 cells }\end{array}$ & $\begin{array}{l}\text { Orally, } 200,400 \mathrm{mg} / \\
\mathrm{kg} \text { for } 21 \text { days }\end{array}$ & $\begin{array}{l}\text { AR: } 62.87 \% \\
\text { TIR: } 57.57 \% \\
\text { Breast Cancer }\end{array}$ & Mitochondrial Pathway & $\begin{array}{l}\text { Bcl-2 } \downarrow ; \text { Bax, Caspase-9, } \\
\text { Caspase- } 7 \uparrow\end{array}$ & $\begin{array}{l}\text { (Xie R.-D. et al., } \\
\text { 2019) }\end{array}$ \\
\hline $\begin{array}{l}\text { Astragalus } \\
\text { mongholicus Bunge } \\
\text { polysaccharide (APS) }\end{array}$ & & $\begin{array}{l}\text { CNE-1, CNE-2, and } \\
\text { SUNE-1 } \\
\text { Mice injected with } \\
\text { CNE-2 cells }\end{array}$ & $\begin{array}{l}\text { 10-80 } \mu \mathrm{g} / \mathrm{ml}, 48 \mathrm{~h} \\
40 \mathrm{mg} / \mathrm{kg} \text { (i.p.) twice a } \\
\text { week for } 4 \text { weeks }\end{array}$ & $\begin{array}{l}\text { AR: Over } 40 \% \\
\text { TIR: Over } 75 \% \\
\text { CV: Under } 50 \% \\
\text { Nasopharyngeal } \\
\text { Carcinoma }\end{array}$ & Mitochondrial Pathway & $\begin{array}{l}\text { Bcl-2\; Bax, Caspase-3, } \\
\text { Caspase- } 9 \uparrow\end{array}$ & $\begin{array}{l}\text { (Zhou Z. et al., } \\
\text { 2017) }\end{array}$ \\
\hline $\begin{array}{l}\text { Astragalus } \\
\text { mongholicus Bunge } \\
\text { cold-water-soluble } \\
\text { polysaccharide (APS4) }\end{array}$ & $\begin{array}{l}\text { Glucose, rhamnose, arabinose, xylose, } \\
\text { mannose, galactose (12.1: } 0.3: 0.6: \\
\text { 1.0:1.0: 1.7.) }\end{array}$ & MGC-803 cells & $0-800 \mu \mathrm{g} / \mathrm{ml}, 24 \mathrm{~h}$ & $\begin{array}{l}\text { AR: } 20.60 \% \\
\text { CV: } 54.23 \% \\
\text { Gastric carcinoma }\end{array}$ & Mitochondrial Pathway & 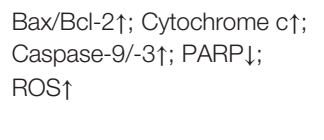 & (Yu et al., 2019) \\
\hline $\begin{array}{l}\text { Aralia elata (Miq.) } \\
\text { Seem. polysaccharide } \\
\text { (AEP-1) }\end{array}$ & $\begin{array}{l}\text { Glucose, galactose, arabinose (6.0: } 3.0 \text { : } \\
\text { 1.0) }\end{array}$ & U-2 OS cell & $\begin{array}{l}25,50 \text {, and } 100 \mu \mathrm{g} / \\
\mathrm{ml}, 24,48 \text {, and } 72 \mathrm{~h}\end{array}$ & $\begin{array}{l}\text { CV: About } 50 \% \\
\text { Osteosarcoma }\end{array}$ & Mitochondrial Pathway & 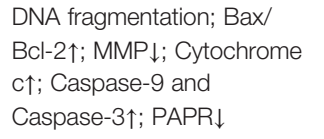 & (Liu et al., 2016b) \\
\hline $\begin{array}{l}\text { Atractylodes } \\
\text { macrocephala Koidz. } \\
\text { polysaccharide (AMPs) }\end{array}$ & & C6 cells & $\begin{array}{l}50,100, \text { and } 200 \mu \mathrm{g} / \\
\mathrm{ml}, 48 \mathrm{~h}\end{array}$ & $\begin{array}{l}\text { CV: } 19.1 \% \\
\text { Glioma }\end{array}$ & Mitochondrial Pathway & $\begin{array}{l}\text { MMP } \downarrow \text {; Cytochrome c } \uparrow ; \\
\text { Capase-3, Caspase-9 } 9 ; \\
\text { PARP } \downarrow\end{array}$ & (Li X. et al., 2014) \\
\hline $\begin{array}{l}\text { Camellia sinensis } \\
\text { polysaccharide (GTP) }\end{array}$ & Glucose & PC cells & $25-100 \mu \mathrm{g} / \mathrm{ml}, 48 \mathrm{~h}$ & $\begin{array}{l}\text { AR: } 73.2 \% \\
\text { CV: About } 50 \% \\
\text { Prostate cancer }\end{array}$ & Mitochondrial Pathway & 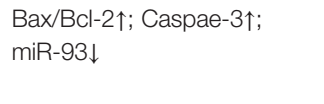 & (Yang et al., 2019) \\
\hline $\begin{array}{l}\text { Citrus } \times \text { aurantiifolia } \\
\text { (Christm.) Swingle } \\
\text { polysaccharide (CAs) }\end{array}$ & $\begin{array}{l}\text { Rhamnose, arabinose, galactose, glucose, } \\
\text { mannose, galacturonic acid (0.67: } 7.67 \text { : } \\
\text { 10.83: 3.83: 4.00: } 1.00)\end{array}$ & $\begin{array}{l}\text { Mice injected with } \\
\text { H22 cells }\end{array}$ & $\begin{array}{l}\text { Orally, } 50 \text { and } \\
250 \mathrm{mg} / \mathrm{kg} \text { for } 3 \\
\text { weeks }\end{array}$ & $\begin{array}{l}\text { TIR: } 58.85 \% \\
\text { Hepatocellular } \\
\text { carcinoma }\end{array}$ & Mitochondrial Pathway & 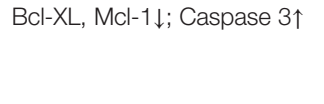 & $\begin{array}{l}\text { (Zhao Y. et al., } \\
\text { 2017) }\end{array}$ \\
\hline $\begin{array}{l}\text { Curcuma kwangsiensis } \\
\text { S.G.Lee \& C.F.Liang } \\
\text { polysaccharides (CKP) }\end{array}$ & $\begin{array}{l}\text { Fructose, xylose, mannose, glucose, } \\
\text { galacose }(25.0,25.0,10.0,12.5,12.5)\end{array}$ & CNE-2 cells & $\begin{array}{l}12.5-100.0 \mu \mathrm{g} / \mathrm{ml}, 48 \\
\mathrm{~h}\end{array}$ & $\begin{array}{l}\text { AR: } 42.85 \% \\
\text { CV: } 76.52 \% \\
\text { Nasopharyngeal }\end{array}$ & Mitochondrial Pathway & 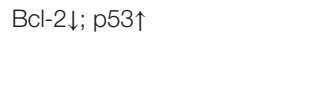 & (Zeng et al., 2012) \\
\hline $\begin{array}{l}\text { Cymbopogon citratus } \\
\text { (DC.) Stapf } \\
\text { polysaccharides (F1 } \\
\text { and F2) }\end{array}$ & $\begin{array}{l}\text { F1; Glucose, Galactose, Xylose, Mannose } \\
(5.1,11.5,74.5,3.2) \\
\text { F2: Glucose, Galactose, Xylose, Mannose } \\
(6.2,9.8,69.5,7.3)\end{array}$ & $\begin{array}{l}\text { Siha and LNCap } \\
\text { cells }\end{array}$ & $\begin{array}{l}100-1,000 \mu \mathrm{g} / \mathrm{mL} \\
24,48 \text { and } 72 \mathrm{~h}\end{array}$ & $\begin{array}{l}\text { CV: Under } 60 \% \\
\text { Reproductive cancer }\end{array}$ & Mitochondrial Pathway & 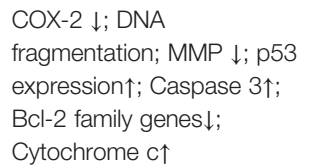 & $\begin{array}{l}\text { (Thangam et al., } \\
\text { 2014) }\end{array}$ \\
\hline
\end{tabular}


Glycyrrhiza inflata

Batalin polysaccharide (GIAP1)

Scleromitrion diffusum (Willd.) R.J.Wang (syn. Hedyotis diffusa Willd) polysaccharide (SDP) Scleromitrion diffusum (Willd.) R.J.Wang (syn. polysaccharides (SDP) Palisada perforata (Bory) K.W.Nam (syn. Laurencia papillosa

(C.Agardh) Greville)

polysaccharide (ASPE)

Paeonia $\times$ suffruticosa Andrews (Peony seed dreg) polysaccharide (CASS)

Punica granatum L.

(Pomegranate) peels

polysaccharide (PPP)

Portulaca oleracea L.

polysaccharide (POL-

P3b)

Cucurbita pepo L.

(Pumpkin)

polysaccharide (PPW)

Pinellia ternata (Thunb.)

Makino polysaccharide

(PTPA)

Carthamus tinctorius L.

(Safflower)

polysaccharide (SPS)

Carthamus tinctorius L.

(Safflower)

polysaccharide (SPS)

Sanguisorba officinalis

L. (Sanguisorbae radix)

polysaccharide

polysaccharide (SRP)
Glucose, galactose, and mannosein $\quad$ SCC-25 cells $\quad 50,100$, and 200 $\mu \mathrm{g} / \quad$ AR:64.07\%

(2.3:1.2:1.2)

$\mathrm{mL}, 48 \mathrm{~h}$

Oral cancer

$$
\text { 1.0) }
$$

A549 cells

Mice injected with

A549 cells

Rhamnose, glucose, galactose, arabinose, Hep2 cells mannose (4.31:4.16:4.49:9.22:27.8)

\section{MDA-MB-231 cells}

25, 100, and 200 $\mu \mathrm{g} / \quad$ AR:66.08\%

$\mathrm{ml}, 48 \mathrm{~h} \quad$ TIR: Nearly $50 \%$

$50,100 \mathrm{mg} / \mathrm{kg}$ for 15 Lung cancer days

25-800 $\mu \mathrm{g} / \mathrm{ml}, 24 \mathrm{~h} \quad$ AR: $67.45 \%$

Larynx squamous

carcinoma

5-100 $\mu \mathrm{g} / \mathrm{ml}, 24 \mathrm{~h} \quad$ AR: $50 \%$

CV: About 20\%

Breast cancer.

L-galactose, L-arabinose, D-glucose

Total sugar, uronic acid, and protein (72.4, U-2 OS cells $19.5,9.7)$

Glucose, galactose

(0.75:1.00)

Galactose, mannose, glucose, arabinose HepG2 cells

(2.02:2.05:1.00:0.52)

50-1,000 $\mu \mathrm{g} / \mathrm{mL}, 24, \quad$ TIR: $50.28 \%$
Pc-3 cells

HCT -116 cells

MCF-7 cells

Hela cells

$\begin{array}{ll}\text { 50-500 } \mu \mathrm{g} / \mathrm{ml}, 48 \mathrm{~h} & \text { AR: } 20.22 \% \text { (Pc-3), } \\ & 17.87 \% \text { (HCT-116), } \\ & 30.94 \% \text { (Hela), } \\ & 38.73 \% \text { (MCF-7) } \\ & \text { Prostate cancer } \\ & \text { Colon cancer } \\ & \text { Breast cancer } \\ & \text { Cervical cancer } \\ \text { 0-400 } \mu \mathrm{g} / \mathrm{ml}, 24-96 \mathrm{~h} & \text { AR: } 49.73 \%\end{array}$

0-400 $\mu \mathrm{g} / \mathrm{ml}, 24-96$ h $\quad$ AR: $49.73 \%$

$$
\text { CV: Over 40\% }
$$

48 , and $72 \mathrm{~h} \quad$ Cervical carcinoma

100, 200 , and $\quad$ AR: $70.3 \%$

$400 \mu \mathrm{g} / \mathrm{ml}, 24,48, \quad \mathrm{CV}$ : Over $30 \%$

and $72 \mathrm{~h}$ Hepatocellular

carcinoma

Cholangiocarcinoma $\quad 25-200 \mu \mathrm{g} / \mathrm{ml}, 24 \mathrm{~h} \quad$ AR: $78.9 \%$

cell lines

CV: Under $50 \%$

Cholangiocarcinoma

MCF cells

$0.04,0.08,0.17$

AR: $54.5 \%$

$0.34,0.68,1.36 \mathrm{mg} / \quad$ TIR: Over $80 \%$

$\mathrm{ml}, 48 \mathrm{~h}$

$\mathrm{HN}-6$ cells

0-1.28 mg/mL, 24- TIR: About 50\%

$72 \mathrm{~h}$

Tongue squamous cell

HL-60 cells

50, 100, 150, 200,

and $400 \mu \mathrm{g} / \mathrm{ml}, 24 \mathrm{~h} \quad \mathrm{CV}$ : Under $50 \%$

Leukemia
Mitochondrial Pathway Bax/Bcl-2 $\downarrow$; MMP $\downarrow$;

Cytochrome c $\uparrow$; Capase-3

and Caspase-9 9 ; PARP $\downarrow$

Mitochondrial Pathway Caspase-9 and $-3 \uparrow$;

Cytochrome $\mathrm{c} \uparrow ; \mathrm{Bax} \uparrow$

Mitochondrial Pathway Caspase-3, Caspase-8, (Wu C. et al.,

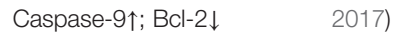

Mitochondrial Pathway Cip1/p21, Kip1/p27个; cyclins D1, D2, E1 transcripts, Cdk2, Cdk4,

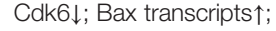

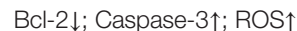

Mitochondrial Pathway mRNA Cyclin A/B1/D1/E1 CDK-1/2/4/6, p15/16/21/

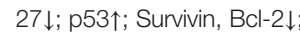
Caspase-3, Caspases-8 and Caspases-91; Cytochrome c, Baxץ; Bcl-

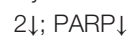

Mitochondrial Pathway $\quad \mathrm{Bax} / \mathrm{Bcl}-2 \uparrow ; \mathrm{MMP} \downarrow$; Cytochrome cศ; Caspase- 9

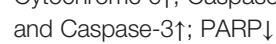

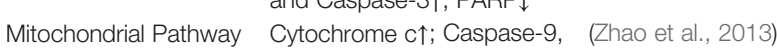

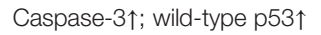

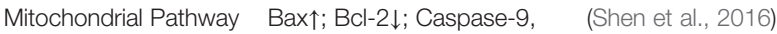

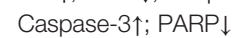

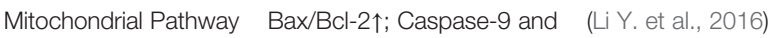

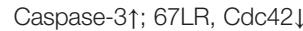

Mitochondrial Pathway $\quad$ Bcl-2 $\downarrow$; MMP-9 $\downarrow$; TIMP $1 \downarrow \quad$ (Luo et al., 2015)

Mitochondrial Pathway $\mathrm{Bcl}-2$, COX-2£; Bax cleaved Caspase- 31

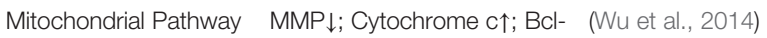
2 protein and mRNAl;

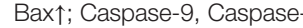




\begin{tabular}{|c|c|c|c|c|c|c|c|}
\hline Drugs & $\begin{array}{l}\text { Monosaccharide } \\
\text { composition/structure }\end{array}$ & Model & Dose and treat time & $\begin{array}{l}\text { Activity and type of } \\
\text { cancer }\end{array}$ & Apoptotic pathway & Involved mechanism & Ref \\
\hline $\begin{array}{l}\text { Sargassum wightii } \\
\text { Greville ex J.Agardh } \\
\text { polysaccharides (SWP) }\end{array}$ & Galactofuranose, arabinose (64.6:11.2) & $\begin{array}{l}\text { MCF7 and MDA- } \\
\text { MB-231 cells }\end{array}$ & $0-500 \mu \mathrm{g} / \mathrm{ml}, 24 \mathrm{~h}$ & Breast cancer & Mitochondrial Pathway & 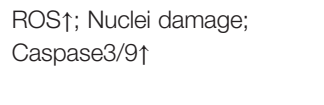 & $\begin{array}{l}\text { (Vaikundamoorthy } \\
\text { et al., 2018) }\end{array}$ \\
\hline $\begin{array}{l}\text { Sargassum } \\
\text { integerrimum Tseng \& } \\
\text { Lu polysaccharide } \\
\text { (SPSa) }\end{array}$ & & Transgenic zebrafish & $\begin{array}{l}0-1.5 \mathrm{mg} / \mathrm{ml} \text { for } 12 \\
\text { and } 24 \mathrm{~h} \\
1,4 \mathrm{mg} / \mathrm{ml} \text { for } 48 \mathrm{~h}\end{array}$ & $\begin{array}{l}\text { AR: } 28.8 \% \\
\text { Lung cancer }\end{array}$ & $\begin{array}{l}\text { Mitochondrial Pathway } \\
\text { G2/M phase arrest }\end{array}$ & 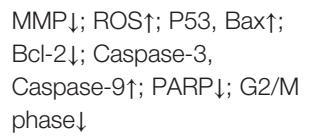 & (Liu et al., 2016a) \\
\hline $\begin{array}{l}\text { Se-containing } \\
\text { polysaccharides from } \\
\text { Ginkgo biloba } L . \text { leaves } \\
\text { (Se-GBLP) }\end{array}$ & 93.7\% carbohydrate & T24 cells & $\begin{array}{l}50-200 \mu \mathrm{g} / \mathrm{ml}, 24,48 \\
\text { or } 72 \mathrm{~h}\end{array}$ & $\begin{array}{l}\text { AR: } 70.3 \% \\
\text { CV: } 30.2 \% \\
\text { Bladder cancer }\end{array}$ & Mitochondrial Pathway & 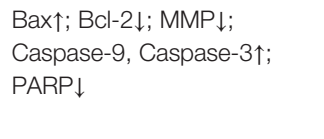 & (Chen et al., 2017) \\
\hline $\begin{array}{l}\text { Pyracantha fortuneana } \\
\text { (Maximowicz) H. L. Li, } \\
\text { J. Arnold Arbor. (Se- } \\
\text { PFPs) }\end{array}$ & $\begin{array}{l}\text { Carbohydrate (93.7\%), uronic acid (2.1\%), } \\
\text { and } 3.7 \mu \mathrm{g} / \mathrm{g} \text { of Se }\end{array}$ & $\begin{array}{l}\text { HEY and SKOV3 } \\
\text { cells } \\
\text { Female mice injected } \\
\text { with HEY cells }\end{array}$ & $\begin{array}{l}0-1,000 \mu \mathrm{g} / \mathrm{ml}, 24 \mathrm{~h} \\
\text { Orally, } 400 \mathrm{mg} / \mathrm{kg} \text { for } \\
28 \text { days }\end{array}$ & $\begin{array}{l}\text { AR: } 41.4 \% \text { (HEY) } \\
\text { AR: } 30.4 \% \text { (SKOV3) } \\
\text { TIR: Over } 40 \% \\
\text { Ovarian cancer }\end{array}$ & Mitochondrial Pathway & 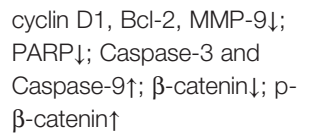 & (Sun et al., 2016) \\
\hline $\begin{array}{l}\text { Millettia pulchra (Voigt) } \\
\text { Kurz (Yulangsan) } \\
\text { polysaccharide } \\
\text { (YLSPS) }\end{array}$ & & $\begin{array}{l}4 \mathrm{~T} 1 \text { cells } \\
\text { Mice injected with } \\
4 \mathrm{~T} 1 \text { cells }\end{array}$ & $\begin{array}{l}10 \% \text { medicated } \\
\text { serum, } 24 \mathrm{~h} \\
150,300,600 \mathrm{mg} / \mathrm{kg} \\
\text { for } 7 \text { days }\end{array}$ & $\begin{array}{l}\text { AR: over 30\% } \\
\text { TIR: over 35\% } \\
\text { Breast Cancer }\end{array}$ & Mitochondrial Pathway & 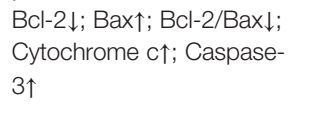 & (Qin et al., 2019) \\
\hline $\begin{array}{l}\text { Boschniakia rossica } \\
\text { (Cham. \& Schltdl.) } \\
\text { B.Fedtsch. } \\
\text { polysaccharide (BRP) }\end{array}$ & & Hep2 cells & $\begin{array}{l}\text { 50, } 100,200,400 \\
\mathrm{mg} / \mathrm{L}, 48 \mathrm{~h}\end{array}$ & $\begin{array}{l}\text { AR:30.9\% } \\
\text { Laryngeal cancer }\end{array}$ & $\begin{array}{l}\text { Mitochondrial Pathway } \\
\text { Death receptors } \\
\text { Pathway }\end{array}$ & 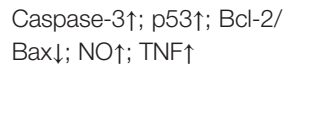 & (Yao et al., 2017) \\
\hline $\begin{array}{l}\text { Boschniakia rossica } \\
\text { (Cham. \& Schltdl.) } \\
\text { B.Fedtsch. } \\
\text { Polysaccharide (BRP) }\end{array}$ & & Hep2 cells & $\begin{array}{l}100,200, \text { and } 400 \\
\mathrm{ug} / \mathrm{ml}, 24 \mathrm{~h}\end{array}$ & $\begin{array}{l}\text { AR: } 60 \% \\
\text { Laryngeal carcinoma }\end{array}$ & $\begin{array}{l}\text { Mitochondrial Pathway } \\
\text { Death receptors } \\
\text { Pathway }\end{array}$ & 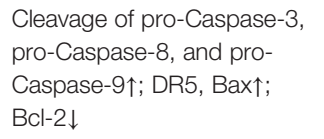 & $\begin{array}{l}\text { Wang et al., } \\
\text { 2014) }\end{array}$ \\
\hline $\begin{array}{l}\text { Zingiber officinale } \\
\text { Roscoe (Ginger) } \\
\text { polysaccharide (GP) }\end{array}$ & $\begin{array}{l}\text { L-rhamnose, D- arabinose, D-mannose, } \\
\text { D-glucose, D-galactose (3.64: } 5.37: 3.04: \\
\text { 61.03: 26.91) }\end{array}$ & HepG2 cells & 0-0.4 mg/ml, $12 \mathrm{~h}$ & $\begin{array}{l}\text { AR: } 16.84 \% \\
\text { CV: } 50.37 \% \\
\text { Hepatocellular } \\
\text { carcinoma }\end{array}$ & $\begin{array}{l}\text { Mitochondrial Pathway } \\
\text { Death receptors } \\
\text { Pathway }\end{array}$ & 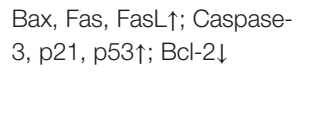 & $\begin{array}{l}\text { (Wang et al., } \\
\text { 2019) }\end{array}$ \\
\hline $\begin{array}{l}\text { Lycium barbarum L. } \\
\text { polysaccharide (LBP) }\end{array}$ & & MLL - ALL cells & $\begin{array}{l}100,500,1,000 \mathrm{mg} / \mathrm{l} \\
12 \mathrm{~h}\end{array}$ & $\begin{array}{l}\text { AR: Over 25\% } \\
\text { Leukemia }\end{array}$ & $\begin{array}{l}\text { Mitochondrial Pathway } \\
\text { Death receptors } \\
\text { Pathway }\end{array}$ & 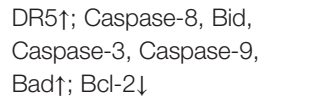 & $\begin{array}{l}\text { (Chen C. et al., } \\
\text { 2019) }\end{array}$ \\
\hline $\begin{array}{l}\text { Astragalus } \\
\text { mongholicus Bunge } \\
\text { polysaccharide (APS) }\end{array}$ & $\begin{array}{l}89.75 \% \text { total carbohydrate } \\
\text { and } 9.3 \% \text { uronic acid }\end{array}$ & MCF-7 cells & $\begin{array}{l}50-1,000 \mu \mathrm{g} / \mathrm{mL}, 24 \\
\mathrm{~h}\end{array}$ & $\begin{array}{l}\text { AR: } 29.7 \% \\
\text { Breast cancer }\end{array}$ & $\begin{array}{l}\text { Death receptors } \\
\text { Pathway }\end{array}$ & $\begin{array}{l}\text { NO and TNF- } \alpha \uparrow ; ~ B a x / B c l- \\
2 \uparrow\end{array}$ & (Li W. et al., 2019) \\
\hline $\begin{array}{l}\text { Gracilariopsis } \\
\text { lemaneiformis (Bory de } \\
\text { Saint-Vincent) } \\
\text { E.Y.Dawson, Acleto \& } \\
\text { Foldvik Polysaccharide } \\
\text { (PGL) }\end{array}$ & $\begin{array}{l}\text { D-galactose and 3,6-anhydro- } L \\
\text {-galactose }(57.38: 41.2)\end{array}$ & A549 cells & 0-100 $\mu \mathrm{g} / \mathrm{ml}, 72 \mathrm{~h}$ & $\begin{array}{l}\text { AR: Over } 5 \% \\
\text { Lung cancer }\end{array}$ & $\begin{array}{l}\text { Death receptors } \\
\text { Pathway }\end{array}$ & Fas/FasL $\uparrow$ & (Kang et al., 2017) \\
\hline $\begin{array}{l}\text { Tussilago farfara } \mathrm{L} \text {. } \\
\text { flower buds } \\
\text { polysaccharide (TFPB1) }\end{array}$ & $\begin{array}{l}\text { Rhamnose, galacturonic acid, glucose, } \\
\text { galactose, and arabinose (13: 13: 1: 7: 12) }\end{array}$ & A549 cells & $\begin{array}{l}31.2-1,000 \mu \mathrm{g} / \mathrm{mL}, \\
48 \mathrm{~h}\end{array}$ & $\begin{array}{l}\text { AR: } 40 \% \\
\text { Lung cancer }\end{array}$ & $\begin{array}{l}\text { Death receptors } \\
\text { Pathway } \\
\text { PI3K/AKT Pathway }\end{array}$ & 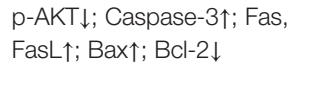 & (Qu et al., 2018) \\
\hline
\end{tabular}




\begin{tabular}{|c|c|c|c|c|c|c|c|}
\hline Drugs & $\begin{array}{l}\text { Monosaccharide } \\
\text { composition/structure }\end{array}$ & Model & Dose and treat time & $\begin{array}{l}\text { Activity and type of } \\
\text { cancer }\end{array}$ & Apoptotic pathway & Involved mechanism & Ref \\
\hline $\begin{array}{l}\text { Carthamus tinctorius L. } \\
\text { (Safflower) } \\
\text { polysaccharide (SPS) }\end{array}$ & & $\begin{array}{l}\text { A549 and YTMLC- } \\
90 \text { cells } \\
\text { Mice injected with } \\
\text { A549 cells }\end{array}$ & $\begin{array}{l}0.04 \text { to } 2.56 \mathrm{mg} / \mathrm{ml} \text {, } \\
24,48 \text {, and } 72 \mathrm{~h} \\
15 \text { to } 135 \mathrm{mg} / \mathrm{kg} \\
\text { (i.p.) for } 30 \text { days }\end{array}$ & $\begin{array}{l}\text { TIR: About } 80 \% \\
\text { Lung cancer }\end{array}$ & $\begin{array}{l}\text { PI3K/AKT Pathway } \\
\text { Death receptors } \\
\text { Pathway } \\
\text { G2/M phase arrest }\end{array}$ & 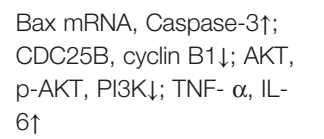 & $\begin{array}{l}(\text { L J. Y.i et al., } \\
\text { 2016) }\end{array}$ \\
\hline $\begin{array}{l}\text { A novel polysaccharide } \\
\text { derived from algae }\end{array}$ & & MCF-7 cells & $1-1,000 \mu \mathrm{g} / \mathrm{ml}, 48 \mathrm{~h}$ & $\begin{array}{l}\text { AR: Over } 16 \% \\
\text { Breast cancer }\end{array}$ & MAPK Pathway & 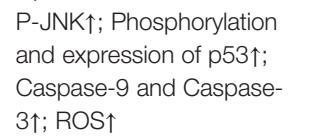 & (Xie et al., 2018) \\
\hline $\begin{array}{l}\text { Astragalus } \\
\text { mongholicus Bunge } \\
\text { polysaccharide (APS) }\end{array}$ & & MG63 cells & $\begin{array}{l}1,5,10,20 \mathrm{mg} / \mathrm{ml} \\
24 \mathrm{~h}\end{array}$ & $\begin{array}{l}\text { AR: About 10\% } \\
\text { Osteosarcoma }\end{array}$ & $\begin{array}{l}\text { MAPK Pathway } \\
\text { (JNK pathway) }\end{array}$ & 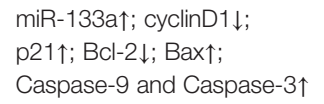 & (Chu et al., 2018) \\
\hline $\begin{array}{l}\text { Astragalus } \\
\text { mongholicus Bunge } \\
\text { polysaccharide (APS) }\end{array}$ & & SKOV3 cells & $\begin{array}{l}50-1,600 \mu \mathrm{g} / \mathrm{ml}, 24 \\
48, \text { and } 72 \mathrm{~h}\end{array}$ & $\begin{array}{l}\text { AR: Over } 40 \% \\
\text { CV: About } 60 \% \\
\text { Ovarian cancer }\end{array}$ & $\begin{array}{l}\text { MAPK Pathway } \\
\text { (JNK pathway) }\end{array}$ & 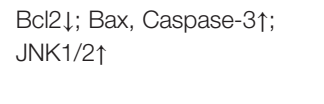 & (Li C. et al., 2018) \\
\hline $\begin{array}{l}\text { Schisandra chinensis } \\
\text { (Turcz.) Baill. } \\
\text { polysaccharide (SCP) }\end{array}$ & & $\begin{array}{l}\text { RCC cell lines (Caki- } \\
\text { 1, Caki-2, and } \\
\text { ACHN) }\end{array}$ & $25-800 \mu \mathrm{g} / \mathrm{ml}, 48 \mathrm{~h}$ & $\begin{array}{l}\text { AR: About } 50 \% \\
\text { CV: Under 50\% } \\
\text { Renal cell carcinoma }\end{array}$ & $\begin{array}{l}\text { MAPK Pathway } \\
\text { Mitochondrial Pathway }\end{array}$ & 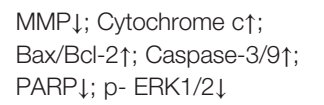 & (Liu et al., 2014) \\
\hline $\begin{array}{l}\text { Aconitum coreanum } \\
\text { (H.Lév.) Rapaics } \\
\text { polysaccharide (CACP) }\end{array}$ & & $\mathrm{H} 22$ cells & $\begin{array}{l}10,20 \text {, and } 40 \mu \mathrm{g} / \mathrm{ml} \\
\text { for } 48 \mathrm{~h}\end{array}$ & $\begin{array}{l}\text { AR: } 43.3 \% \\
\text { TIR: } 45.9 \% \\
\text { Hepatocellular } \\
\text { carcinoma }\end{array}$ & $\begin{array}{l}\text { PI3K/AKT Pathway } \\
\text { MAPK Pathway }\end{array}$ & $\begin{array}{l}\text { PTTG1 mRNA } \downarrow ; \text { p-AKT } \downarrow ; \text { p- } \\
\text { p38MARK } \uparrow\end{array}$ & (Liang et al., 2015) \\
\hline $\begin{array}{l}\text { Astragalus } \\
\text { mongholicus Bunge } \\
\text { polysaccharide (APS) }\end{array}$ & & $\begin{array}{l}\text { ASPC-1, PANC-1 } \\
\text { cells }\end{array}$ & $50-400 \mu \mathrm{g} / \mathrm{ml}, 24 \mathrm{~h}$ & $\begin{array}{l}\text { RA: Over 30\% (ASPC- } \\
\text { 1) } \\
\text { Over 20\% (PANC-1) } \\
\text { Pancreatic cancer }\end{array}$ & $\begin{array}{l}\text { MAPK Pathway } \\
\text { PI3K/AKT Pathway }\end{array}$ & 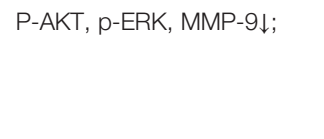 & (Wu et al., 2018a) \\
\hline $\begin{array}{l}\text { Panax ginseng } \\
\text { C.A.Mey. } \\
\text { polysaccharide (GPS) }\end{array}$ & & MG-63 cell & $\begin{array}{l}20,10,5 \text {, and } 1 \mathrm{mM}, \\
24 \mathrm{~h}\end{array}$ & $\begin{array}{l}\text { AR: } 57 \% \\
\text { Osteosarcoma }\end{array}$ & $\begin{array}{l}\text { MAPK Pathway } \\
\text { PI3K/AKT Pathway }\end{array}$ & 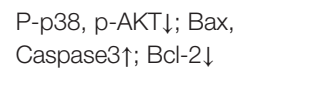 & $\begin{array}{l}\text { (Zhang X. et al., } \\
\text { 2017) }\end{array}$ \\
\hline $\begin{array}{l}\text { Gracilariopsis } \\
\text { lemaneiformis (Bory de } \\
\text { Saint-Vincent) } \\
\text { E.Y.Dawson, Acleto \& } \\
\text { Foldvik polysaccharide } \\
\text { selenium nanoparticles } \\
\text { (GLP-SeNPs) }\end{array}$ & & U87 cells & $\begin{array}{l}0.2,1,5 \text {, and } 10 \mathrm{mg} / \\
\mathrm{ml}, 72 \mathrm{~h}\end{array}$ & $\begin{array}{l}\text { CV: } 44.6 \% \\
\text { Glioma }\end{array}$ & $\begin{array}{l}\text { MAPK Pathway } \\
\text { PI3K/AKT Pathway }\end{array}$ & 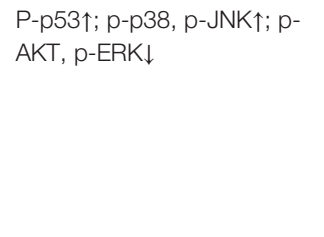 & (Jiang et al., 2014) \\
\hline $\begin{array}{l}\text { Hordeum vulgare } L \text {. } \\
\text { polysaccharide (BP-1) }\end{array}$ & $\begin{array}{l}\text { Glucose, xylose, arabinose, rhamnose } \\
(8.82: 1.92: 1.50: 1.00)\end{array}$ & HT-29 cells & $0-150 \mu \mathrm{g} / \mathrm{ml}, 48 \mathrm{~h}$ & $\begin{array}{l}\text { AR: } 49.17 \% \\
\text { Colon cancer }\end{array}$ & $\begin{array}{l}\text { MAPK Pathway } \\
\text { NF-kB Pathway }\end{array}$ & 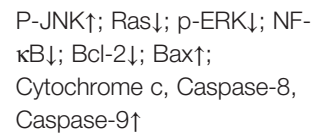 & $\begin{array}{l}\text { (Cheng et al., } \\
\text { 2016) }\end{array}$ \\
\hline $\begin{array}{l}\text { Astragalus } \\
\text { mongholicus Bunge } \\
\text { polysaccharide (APS) } \\
\text { and Apatinib }\end{array}$ & & AGS cells & $0-600 \mu \mathrm{g} / \mathrm{ml}, 24 \mathrm{~h}$ & $\begin{array}{l}\text { AR: Over 20\% } \\
\text { TIR: Over60\% } \\
\text { Gastric cancer }\end{array}$ & PI3K/AKT Pathway & p-AKT $\downarrow ;$ MMP-9 $\downarrow$ & (Wu et al., 2018b) \\
\hline $\begin{array}{l}\text { Capsosiphon } \\
\text { fulvescens (C.Agardh) } \\
\text { Setchell \& N.L.Gardner } \\
\text { polysaccharide (Cf-PS) }\end{array}$ & Xylose, mannose (85:15) & AGS cells & $0-5 \mathrm{mg} / \mathrm{ml}, 24 \mathrm{~h}$ & $\begin{array}{l}\text { CV: About } 40 \% \\
\text { Gastric cancer }\end{array}$ & PI3K/AKT Pathway & 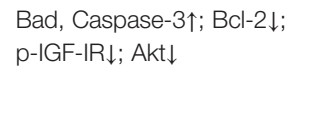 & $\begin{array}{l}\text { (Kwon and Nam, } \\
\text { 2007) }\end{array}$ \\
\hline
\end{tabular}

C.A.Mey

polysaccharide (GPS)

lemaneiformis (Bory de

Saint-Vincent)

E.Y.Dawson, Acleto \&

Foldvik polysaccharide

selenium nanoparticles

0.04 to $2.56 \mathrm{mg} / \mathrm{ml}, \quad$ TIR: About $80 \%$

p.) for 30 days

MG63 cells

$1,5,10,20 \mathrm{mg} / \mathrm{ml}$,

$50-1,600 \mu \mathrm{g} / \mathrm{ml}, 24$

RCC cell lines (Cak-

1, Caki-2, an

$\mathrm{ACHN})$

H22 cells

ASPC-1, PANC-1
cells

MG-63 cell

$$
24
$$

$0.2,1,5$, and $10 \mathrm{mg} / \mathrm{CV}: 44.6 \%$ 


\begin{tabular}{|c|c|c|c|c|c|c|c|}
\hline Drugs & $\begin{array}{l}\text { Monosaccharide } \\
\text { composition/structure }\end{array}$ & Model & Dose and treat time & $\begin{array}{l}\text { Activity and type of } \\
\text { cancer }\end{array}$ & Apoptotic pathway & Involved mechanism & Ref \\
\hline $\begin{array}{l}\text { Cornus officinalis } \\
\text { Siebold \& Zucc. } \\
\text { polysaccharide } \\
\text { polysaccharide (COP) }\end{array}$ & & HepG2 cells & $\begin{array}{l}6.25,12.5, \text { and } 25 \\
\mathrm{mg} / \mathrm{ml}, 72 \mathrm{~h}\end{array}$ & $\begin{array}{l}\text { AR: } 20.1 \% \\
\text { Hepatocellular } \\
\text { carcinoma }\end{array}$ & PI3K/AKT Pathway & 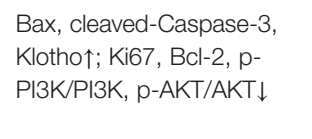 & (Li and Sun, 2019) \\
\hline $\begin{array}{l}\text { Dipsacus asperoides } \\
\text { C.Y.Cheng \& } \\
\text { T.M.Aiwater-soluble } \\
\text { polysaccharide } \\
\text { (ADAPW) }\end{array}$ & $\begin{array}{l}\text { Glucose, rhamnose, arabinose mannose } \\
(8.54: 1.83: 1.04: 0.42 .)\end{array}$ & HOS cells & $\begin{array}{l}100,200, \text { and } \\
400 \mu \mathrm{g} / \mathrm{ml}, 24 \mathrm{~h}\end{array}$ & $\begin{array}{l}\text { AR: } 55.3 \% \\
\text { Osteosarcoma }\end{array}$ & PI3K/AKT Pathway & 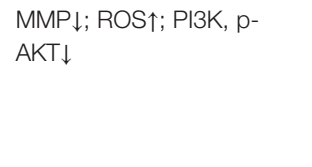 & $\begin{array}{l}\text { (Chen et al., } \\
\text { 2013b) }\end{array}$ \\
\hline $\begin{array}{l}\text { Glycyrrhiza inflata } \\
\text { Batalin polysaccharide } \\
\text { (GPSa) }\end{array}$ & & $\begin{array}{l}\text { Mice injected with } \\
\text { H22 cells } \\
\text { H22 and BEL7402 } \\
\text { cells }\end{array}$ & $\begin{array}{l}\text { Orally, } 25,50 \text {, and } \\
75 \mathrm{mg} / \mathrm{kg} \text { for } 10 \text { days } \\
2-1,250 \mu \mathrm{g} / \mathrm{ml}, 24 \text {, } \\
48 \mathrm{~h}\end{array}$ & $\begin{array}{l}\text { TIR: Over } 85 \% \\
\text { Hepatocellular } \\
\text { carcinoma }\end{array}$ & PI3K/AKT Pathway & $\begin{array}{l}\text { P53 DNA and protein } \uparrow ; p- \\
\text { PI3K and p-AKT } \downarrow\end{array}$ & $\begin{array}{l}\text { (Chen et al., } \\
\text { 2013a) }\end{array}$ \\
\hline $\begin{array}{l}\text { Scutellaria barbata } \\
\text { D.Don water-soluble } \\
\text { polysaccharide } \\
\text { (SPS2p) }\end{array}$ & $\begin{array}{l}\text { Arabinose, mannose, glucose, galactose } \\
(1.31: 1.00: 3.59: 1.59)\end{array}$ & HT29 cells & $\begin{array}{l}10,20,40 \text {, and } 80 \\
\mathrm{mg} / \mathrm{l}, 24 \mathrm{~h}\end{array}$ & $\begin{array}{l}\text { AR: Over } 30 \% \\
\text { Colon cancer }\end{array}$ & PI3K/AKT Pathway & 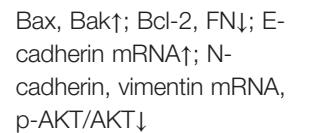 & (Sun et al., 2017) \\
\hline $\begin{array}{l}\text { Aster tataricus L.f. } \\
\text { polysaccharide (ATP-II) }\end{array}$ & $\begin{array}{l}\text { Glucose, galactose, mannose, rhamnose, } \\
\text { and arabinose (2.1:5.2:2.1:1.0:1.2) }\end{array}$ & $\begin{array}{l}\text { C6 cells } \\
\text { SD rats injected with } \\
\text { C6 cells }\end{array}$ & $\begin{array}{l}50-1,000 \mu \mathrm{g} / \mathrm{ml}, 24, \\
48, \text { and } 72 \mathrm{~h} \\
\text { Orally, } 50,100 \text {, and } \\
200 \mathrm{mg} / \mathrm{kg} \text { for } 2 \\
\text { weeks }\end{array}$ & Glioma & $\begin{array}{l}\text { PI3K/AKT Pathway } \\
\text { Mitochondrial Pathway }\end{array}$ & 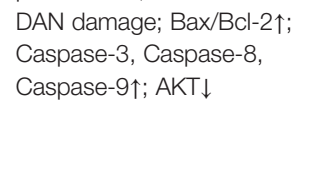 & (Du et al., 2014) \\
\hline $\begin{array}{l}\text { Angelica sinensis (Oliv.) } \\
\text { Diels polysaccharide } \\
\text { (ASP) }\end{array}$ & & SH-SY5Y cells & $0-500 \mu \mathrm{g} / \mathrm{ml}, 48 \mathrm{~h}$ & $\begin{array}{l}\text { AR: Over } 10 \% \\
\text { CV: Under } 30 \% \\
\text { Neuroblastoma }\end{array}$ & $\begin{array}{l}\text { PI3K/AKT Pathway } \\
\text { JAK/STAT Pathway }\end{array}$ & 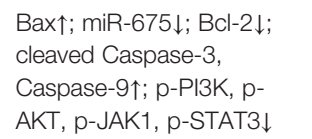 & (Yang et al., 2018) \\
\hline $\begin{array}{l}\text { Portulaca oleracea L. } \\
\text { polysaccharide (POL- } \\
\text { P3b) }\end{array}$ & & HeLa cells & $\begin{array}{l}100 \text { and } 200 \mu \mathrm{g} / \mathrm{ml} \text {, } \\
24 \mathrm{~h}\end{array}$ & $\begin{array}{l}\text { AR: About } 0.06 \\
\text { Cervical cancer }\end{array}$ & TLR4/NF-kB Pathway & 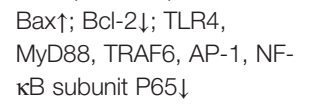 & $\begin{array}{l}\text { (Zhao R. et al., } \\
\text { 2017) }\end{array}$ \\
\hline $\begin{array}{l}\text { Polygala tenuifolia } \\
\text { Willd. polysaccharide } \\
\text { (PTP) }\end{array}$ & & OVCAR-3 cells & $\begin{array}{l}0.1-1.6 \mathrm{mg} / \mathrm{mL}, 24 \\
48,72 \mathrm{~h}\end{array}$ & $\begin{array}{l}\text { AR: } 75.6 \% \\
\text { Ovarian cancer }\end{array}$ & $\begin{array}{l}\text { NF-кB Pathway } \\
\text { Mitochondrial Pathway }\end{array}$ & 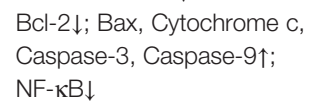 & $\begin{array}{l}\text { (Zhang et al., } \\
\text { 2015) }\end{array}$ \\
\hline $\begin{array}{l}\text { Angelica sinensis (Oliv.) } \\
\text { Diels polysaccharide }\end{array}$ & & T47D cells & $\begin{array}{l}12.5,25,50, \text { and } \\
100 \mu \mathrm{g} / \mathrm{ml}, 24 \mathrm{~h}\end{array}$ & Breast cancer & CREB Pathway & CREB $\uparrow ; \mathrm{Bax} \uparrow ; \mathrm{Mcl}-1 \downarrow$ & (Zhou et al., 2015) \\
\hline (ASP) & & $\begin{array}{l}\text { Mice injected with } \\
\text { T47D cells }\end{array}$ & $\begin{array}{l}0.2 \mathrm{mg} / \mathrm{kg} \text { (i.p.) for } 4 \\
\text { weeks }\end{array}$ & & & & \\
\hline $\begin{array}{l}\text { Astragalus } \\
\text { mongholicus Bunge } \\
\text { polysaccharide (APS) }\end{array}$ & & $\begin{array}{l}\text { Hepatocellular } \\
\text { carcinoma cells }\end{array}$ & $\begin{array}{l}0.1,0.5,1 \mathrm{mg} / \mathrm{ml}, 3 \\
\text { days }\end{array}$ & $\begin{array}{l}\text { AR: Over } 10 \% \\
\text { Hepatocellular } \\
\text { carcinoma }\end{array}$ & Notch Pathway & 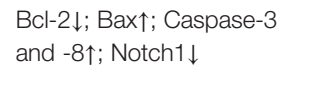 & $\begin{array}{l}\text { (Huang et al., } \\
\text { 2016) }\end{array}$ \\
\hline $\begin{array}{l}\text { Cucurbita pepo L. } \\
\text { (Pumpkin) } \\
\text { polysaccharide (PPPF) }\end{array}$ & $\begin{array}{l}\text { Galactose, mannose, glucose, arabinose } \\
(2.02: 2.05: 1.00: 0.52)\end{array}$ & $\begin{array}{l}\text { HepG2 cells } \\
\text { Female mice injected } \\
\text { with HepG2 cells }\end{array}$ & $\begin{array}{l}200,400 \mu \mathrm{g} / \mathrm{ml}, 48 \mathrm{~h} \\
0.25,0.5, \text { and } 1.0 \\
\mathrm{mg} / \mathrm{kg} \text { (i.p.) for } 18 \\
\text { days }\end{array}$ & $\begin{array}{l}\text { AR: } 71.5 \% \\
\text { TIR: } 57.56 \% \\
\text { CV: } 28.7 \% \\
\text { Hepatocellular } \\
\text { carcinoma }\end{array}$ & JAK/STAT Pathways & 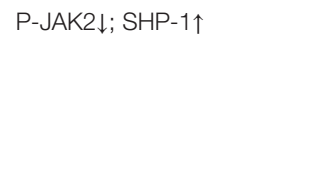 & (Shen et al., 2017) \\
\hline $\begin{array}{l}\text { Panax ginseng } \\
\text { C.A.Mey. }\end{array}$ & $\begin{array}{l}\text { Galactose, arabinose, glucose, } \\
\text { galacturonic acid (3.7:1.6:0.5:5.4) }\end{array}$ & HGC-27 cells & $\begin{array}{l}100,200,400 \mu \mathrm{g} / \mathrm{ml}, \\
48 \mathrm{~h}\end{array}$ & $\begin{array}{l}\text { AR: } 65.0 \% \\
\text { CV: Under } 50 \% \\
\text { Gastric cancer }\end{array}$ & $\begin{array}{l}\text { Mitochondrial Pathway } \\
\text { Death receptors } \\
\text { Pathway }\end{array}$ & 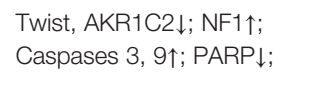 & (Li C. et al., 2014) \\
\hline
\end{tabular}




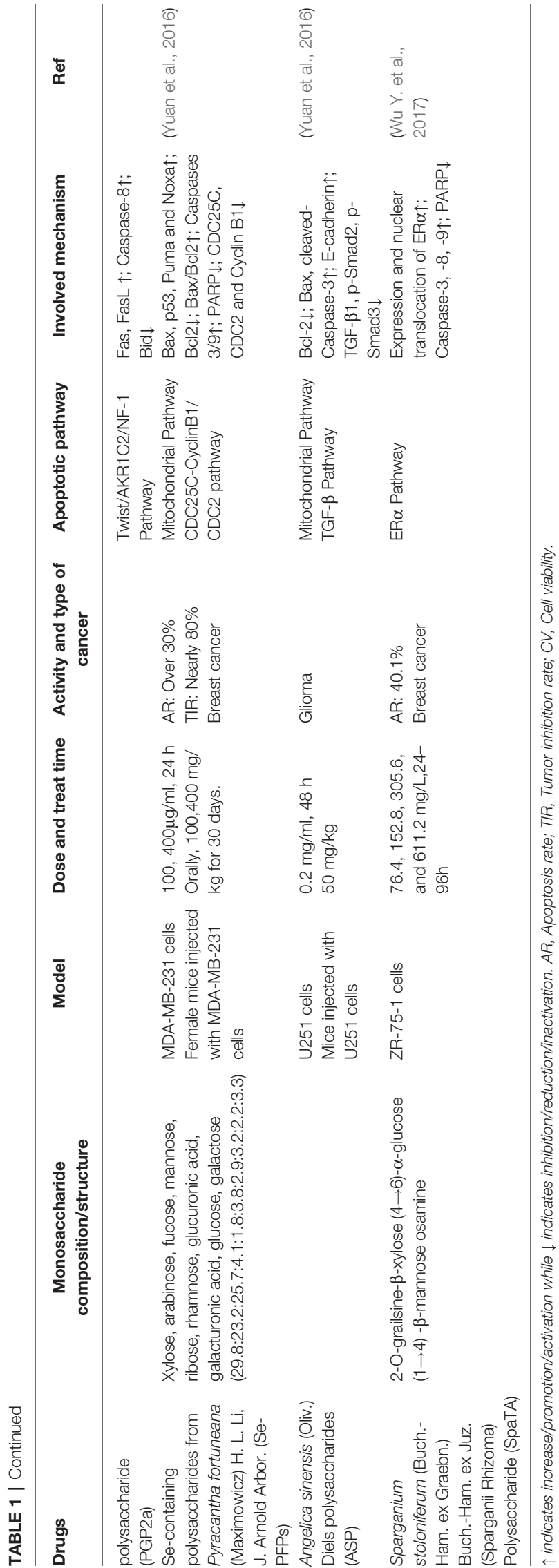

2015). Stimulation of Bcl-2 homology 3 (BH3) proteins such as Bim, Bid, Bad, Puma, Noxa, may transiently interact with Bax or $\mathrm{Bak}$, resulting in the inactivation of $\mathrm{Bcl}-2$ and $\mathrm{Bcl}-\mathrm{XL}$ and conformational changes of Bax and Bak (Prenek et al., 2017). Activated Bax and Bak can form higher-order homopolymers and stably insert into the outer membrane of mitochondria, which leads to the loss of MMP and promotes the formation of MOMP (Zhou X. et al., 2017; Heinicke et al., 2018). MOMP further promotes the release of cytochrome $\mathrm{c}$ into the cytoplasm, which is considered to be a key factor in apoptosis. Once cytochrome $c$ is released, it and Apaf- 1 co-activate the initiator Caspase and activate the end effector Caspase to induce apoptosis (Huang et al., 2015). A study in 2015 reported that after the treatment of Millettia pulchra (Voigt) Kurz (Yulangshan) polysaccharide (YLSPS), the apoptosis index of 4T1 cells obviously increased, and the primary breast cancer tumors significantly regressed. Related mechanisms have also been revealed, namely that YLSPS could induce mitochondrialdependent apoptosis by reducing Bcl-2 levels and increasing Bax levels, releasing cytochrome c, and activating caspase-3 (Qin et al., 2019). An in vivo investigation showed that Astragalus membranaceus Fisch. ex Bunge polysaccharide (APS) inhibited $57.57 \%$ of breast cancer tumors by reducing the expression of Bcl-2 protein and up-regulating the expression of Bax, Caspase9, and Caspase-7. Consequently, this study confirmed the effectiveness of APS in apoptosis regulation (Xie R.-D. et al., 2019). Glycyrrhiza inflata Batalin polysaccharide (GIAP1) dosedependently inhibited the proliferation of SCC-25 cells via inducing apoptosis. The relevant mechanism was related to reduce $\mathrm{Bax} / \mathrm{Bcl}-2$ ratio, disrupt the MMP, and cause the release of cytochrome $\mathrm{c}$ to cytosol. Besides, GIAP1 triggered activation of capase- 3 and Caspase- 9 , as well as the degradation of PARP (Zeng et al., 2015).

In normal cells, ROS can be promptly eliminated by antioxidants such as glutathione, superoxide dismutase and peroxidase, maintained at low levels, and harmless (Dong et al., 2019). However, in tumor cells, ROS production is excessive and cannot be cleared in time, leading to oxidative stress (Dong et al., 2019). If further stimulation is given to increase the production of ROS, it will aggravate oxidative stress and participate in mitochondrial pathway to induce apoptosis (Li et al., 2017a; Cho et al., 2018; Sun et al., 2018). It has been demonstrated that an alkaline polysaccharide (ADAPW) from Dipsacus asperoides C.Y.Cheng \& T.M.Ai showed apoptosis in human osteosarcoma cell line HOS cells. This was because ADAPW caused a considerable intracellular ROS production, resulting in a remarkable change in MMP (Chen et al., 2013b). APS4, a novel cold-water-soluble polysaccharide was isolated from Astragalus membranaceus Fisch. ex Bunge, which had the potential to induce human gastric cancer MGC-803 cell apoptosis. APS4 significantly generated a large amount of ROS and then caused the collapse of MMP, the increase of the pro-apoptotic/anti-apoptotic (Bax/ Bcl-2) ratios, ultimately the cleavage of PARP (Yu et al., 2019). Similarly, Sargassum wightii Greville ex J.Agardh polysaccharides induced the apoptosis in the breast cancer cells 
by increasing ROS generation, cleaving mitochondrial membrane and nuclei damage (Vaikundamoorthy et al., 2018).

P53 induces apoptosis mainly through the mitochondrial pathway (Zhang S. et al., 2019). The ability to induce apoptosis may depend on the transcription of the gene and may not be related to transcription (Zhao et al., 2012). Activated p53 moves to the nucleus and regulates the transcription of these proapoptotic genes such as Puma, Bax, Bak, Noxa and Bid (Zhao et al., 2012; Yamada and Yoshida, 2019). Additionally, at the beginning of apoptosis, p53 can directly interact with proapoptotic factors in mitochondria, activating Bax and/or Bak (Zhao et al., 2012; Yamada and Yoshida, 2019).Se-containing polysaccharides from Pyracantha fortuneana (Maxim.) H.L.Li (Se-PFPs) had required potential to combat breast cancer. It has been indicated that Se-PFP could inhibit $80 \%$ of breast cancer tumors by inducing apoptosis via promoting the expression of p53 and the further increasing Bax, Puma, and Noxa, decreasing $\mathrm{Bcl} 2$, and increasing Caspase-3 activity in MDA-MB-231 cells (Yuan et al., 2016). Similarly, it has been reported that peony seed dreg polysaccharides (CASS) triggered apoptosis in cervical cancer Hela cells and this was closely related to the accumulation of P53 by CASS, leading to the release of downstream mitochondrial factors cytochrome $c$ to cytosol, the activation of initiator Caspases- 8 and -9 , and subsequent the cleavage of Caspase-3 (Zhang F. et al., 2017). The expression of p53 was significantly increased in the treatment of Cymbopogon citratus (DC.) Stapf polysaccharide, which down-regulated the expression of anti-apoptotic factor Bcl-2, up-regulated the proapoptotic factor Bax, and induced cell DNA fragmentation. In addition, it could also up-regulate Caspase 3, down-regulate Bcl2 family genes and promote the release of cytochrome $c$, and finally successfully achieved the induction of apoptosis to regulate reproductive cancer (Thangam et al., 2014).

Of course, there are many plant polysaccharides that could induce apoptosis of various cancer cells through the mitochondrial pathway similar to the above. The specific mechanism is shown in Figure $\mathbf{1}$ and Table $\mathbf{1 .}$

\section{Death Receptor Pathway}

Activate death receptors and trigger the family of TNF, including TNF-R1, Fas (also known as CD95 or Apo-1 TNFRSF6), TRAIL receptors (TRAIL-R1, TRAIL-R2, also known as DR4 and DR5), DR3 and DR6, are also common apoptosis pathways (Zhou X. et al., 2017). These receptors are stimulated and involved by the corresponding ligand, leading to the aggregation of receptors, the recruitment of FADD, and subsequent accumulation of proCaspase-8, which in turn activates Caspase- 8 (Huang et al., 2018). Activated Caspase- 8 can directly activate Caspase- $3,-7$ and play a pro-apoptotic effect (Liang et al., 2018). Caspase- 8 can also induce MOMP and trigger the release of cytochrome $c$ by cleaving Bid, initiating the effect of Caspase activation and apoptosis (Zhou X. et al., 2017). Figure 2 depicts the proapoptotic activity of plant polysaccharides via death receptor pathway. Several plant polysaccharides have been found to affect death receptors. The anti-laryngeal cancer activity of Boschniakia rossica (Cham. \& Schltdl.) B.Fedtsch. polysaccharide (BRP) was the result of its induction of apoptosis in Hep2 cells through death receptor-mediated pathway. The specific mechanism is that BRP promoted nitric oxide (NO) production and increased the secretion of TNF (Yao et al., 2017), and also increased the expression of death receptor DR5 and promoted pro-Caspase-3, pro-Caspase-8, pro-Caspase- 9 and Bax cleavage, and reduced Bcl-2 (Wang et al., 2014). Qu et al. found that a polysaccharide, TFPB1, from the flower buds of Tussilago farfara L. exerted a pro-apoptotic effect in human lung cancer A549 cells, while upregulating the expression of Fas, FasL, and Bax, and downregulating the expression of $\mathrm{Bcl}-2$ is one of the reasons ( $\mathrm{Qu}$ et al., 2018). Death receptor-mediated extrinsic apoptotic pathway was involved in the Panax ginseng C.A.Mey. polysaccharide (PGP2a)-induced apoptosis in HGC-27 cells, which was manifested in that PGP2a increased significantly the expression of Fas and FasL, further promoted the cleavage of pro-Caspase-8, and activated Caspase- 9 and Caspase-3 (Li C. et al., 2014). Ginger polysaccharide (GP), which consists of L-rhamnose, Darabinose, $\mathrm{D}$-mannose, $\mathrm{D}$-glucose, and $\mathrm{D}$-galactose in a molar ratio of 3.64: 5.37: 3.04: 61.03: 26.91, has been shown that have good effects in the induction of hepatocellular carcinoma HepG2 cells apoptosis. This may be related to up-regulate the expression of Bax, Fas, FasL, Caspase-3, p21, and p53, and down-regulate the expression of Bcl-2 (Wang et al., 2019). In lung cancer A549 cells, Gracilariopsis lemaneiformis (Bory de Saint-Vincent) E.Y.Dawson, Acleto \& Foldvik polysaccharide (PGL) induced apoptosis by activating genes involved in the death receptor apoptotic pathway and that the Fas/FasL signaling pathway might play a critical role (Kang et al., 2017). Interestingly, Carthamus tinctorius L. polysaccharide (SPS) injection also inhibited $80 \%$ of lung cancer tumor growth in mice, probably due to its positive effect on TNF- $\alpha$ and IL-6 expression (Li J. Y. et al., 2016). Combination medication is often an effective way to treat disease. Chen et al. studied the effect of combination of Lycium barbarum L. polysaccharide (LBP) and TRAIL on MLL rearranged leukemic cells. The results showed that a significant increase in the expression level of DR5 receptor, Caspase-8, Caspase-3, Caspase-9, and Bad, indicating that LBP and TRAIL can further increase the sensitivity of MLL-ALL cell lines to TRAIL-induced apoptosis by increasing the expression level of DR5 receptors on cells (Chen C. et al., 2019).

\section{The Regulation of Signal Conduction}

Apoptosis can be induced not only directly through the mitochondrial pathway and death receptor pathway, but also by affecting their upstream signal transduction (Wei et al., 2011). Plant polysaccharides have been found that they could affect some upstream signal transduction such as MAPK Pathway, PI3K/AKT Pathway, NF- $\mathrm{KB}$ Pathway, as shown in Figure 3 and Table 1.

\section{MAPK Pathway}

Many of the signaling pathways that regulate cancer cell apoptosis are members of the MAPKs family (Papa et al., 2019). Extracellular signal-regulated kinase (ERK), c-Jun Nterminal kinase (JNK), and p38 kinase are three well-defined MAPK subfamilies in mammals (Petrache et al., 1999). JNK promotes apoptosis through two different mechanisms. The 
translocation of activated JNK to the nucleus increases the expression of pro-apoptotic genes by activating c-Jun/AP1 dependent mechanisms (Dhanasekaran and Reddy, 2008). On the other hand, the activated JNK translocates to mitochondria and phosphorylates $\mathrm{BH} 3$ protein, thereby antagonizing $\mathrm{Bcl}-2$ and Bcl-XL, and finally exert anti-apoptotic activity (Iurlaro and Muñoz-Pinedo, 2016). ERK is one of the anti-apoptotic members. ERK is usually deregulated in tumors due to mutations in Ras or B-Raf. It mainly exerts a anti-apoptotic effect by promoting the activity of anti-apoptotic proteins such as Bcl-2, Bcl-XL, Mcl-1, IAP, and inhibiting apoptotic proteins such as Bad and Bim (Westhoff and Fulda, 2009; Cagnol and Chambard, 2010). While p38 MAPK-dependent apoptosis is mediated by STAT1, CHOP, FAK, SMAD, cytochrome c, NF$\kappa \mathrm{B}, \mathrm{PTEN}$, and $\mathrm{p} 53$-mediated downstream events (Yokota and Wang, 2016). SCP, a polysaccharide from Schisandra chinensis (Turcz.) Baill., showed high cytotoxic potential in Caki-1 cells by inducing apoptosis, which was related to inhibit ERK1/2 phosphorylation and further induce apoptosis in the mitochondrial pathway (Liu et al., 2014). Aconitum coreanum (H.Lév.) Rapaics polysaccharide (CACP) could significantly inhibit the growth of hepatocellular carcinoma cells, and its tumor suppression rate could reach $45.9 \%$, which is the result of CACP treatment activating the p-p38 MARK-dependent apoptosis pathway (Liang et al., 2015). It has been reported $G$. lemaneiformis polysaccharide-Nano-selenium particles (GLPSeNPs) had high selectivity between normal cells and cancer cells, and could effectively and safely fight cancer. Its anticancer effect was mainly dependent on the MAPKs pathway. GLPSeNPs were capable of upregulating the phosphorylation of proapoptotic kinases p38 and JNK in a dose-dependent trend, however, remarkably repressing the phosphorylation of antiapoptotic kinases AKT and ERK (Jiang et al., 2014). Cisplatin is one of common chemotherapy drugs that can kill cancer cells by mediating apoptosis. Interestingly, APS could increase the sensitivity of ovarian cancer SKOV3 cells to cisplatin, which may be related to APS activating JNK1/2 signalling pathway (Li C. et al., 2018).

\section{PI3K/AKT Pathway}

$\mathrm{PI} 3 \mathrm{~K} / \mathrm{AKT}$ is involved in regulating cell proliferation, apoptosis and cell cycle, and is an anti-apoptotic pathway worth exploring (Franke et al., 2003; Liu et al., 2019). In tumor cells the PI3K/ AKT signaling pathway is mostly activated (Xie et al., 2017). Activated AKT directly phosphorylates Ser136 of Bad, which binds to the 14-3-3 protein, promotes Bcl-2/Bcl-XL expression, thereby inhibiting apoptosis through the mitochondrial pathway (Chang et al., 2003; Arlt et al., 2013). In addition, AKT can inhibit apoptosis by activating NF-KB (Chang et al., 2003). Blocking this pathway can effectively promote cancer cell apoptosis to exert anti-cancer effect. SPS2p is a Scutellaria barbata D.Don polysaccharide that could inhibit the activation of the PI3K/AKT signaling pathway in colon cancer cells. This was demonstrated by the fact that SPS2p could promote HT29 cell apoptosis via down-regulating the ratio of p-AKT/AKT, and the expression of Bcl-2 (Sun et al., 2017). It was found that Aster tataricus L.f. polysaccharide (ATP-II) induced apoptosis in glioma cells by reducing the PI3K/AKT signaling pathway. Specifically, ATP-II down-regulated p-AKT protein expression and regulated the ratio of AKT-mediated pro-apoptotic and antiapoptotic protein Bax/Bcl-2 (Du et al., 2014). Chen et al. studied the antitumor effect of $G$. inflata polysaccharide (GPSa) on human hepatocellular carcinoma cells and its mechanism, and it was found that GPSa had good anticancer activity in vitro and in vivo. The possible mechanism was that it promoted the apoptosis of $\mathrm{H} 22$ cells by influencing the P53/PI3K/AKT pathway (Chen et al., 2013a). In addition, Kwon and Nam found that Capsosiphon fulvescens (C.Agardh) Setchell \& N.L.Gardner polysaccharide (Cf-PS) stimulated AGS cells significantly decreased Bcl-2 expression, activated Caspase-3, inactivated the PI3K/AKT pathway, and showed anti-gastric cancer activity (Kwon and Nam, 2007). In 2016, Li et al. reported that SPS inhibited tumor growth by reducing the expression of AKT, p-AKT, and PI3K and increasing mRNA levels of Bax and Caspase-3 (Li J. Y. et al., 2016). In hepatocellular carcinoma HepG2 cells, Cornus officinalis Siebold \& Zucc. polysaccharide could promote the expression of apoptosis-related proteins Bax and Caspase-3, and inhibit the expression of $\mathrm{Bcl}-2$ and $\mathrm{PI} 3 \mathrm{~K} / \mathrm{AKT}$ pathway-related proteins, thus showing pro-apoptotic activity (Li and Sun, 2019). In the same way, CACP could promote apoptosis by inactivating the P13K/AKT signaling pathway and inhibit H22 cell growth in vitro and in vivo (Liang et al., 2015).

\section{NF- $\kappa B$ Pathway}

$\mathrm{NF}-\kappa \mathrm{B}$ is one of the key factors controlling the anti-apoptotic response (Namba et al., 2007). In the cytoplasm, NF- KB (p50/ $\mathrm{p} 65$ ) and $\mathrm{lkB}$ combine to form a trimer, and p50/p65 is unable to undergo nuclear translocation in the resting state of cell ( $\mathrm{Yu}$ et al., 2017). When cells are stimulated by specific external signals, IKK $\beta$ subunit of IKK is activated by phosphorylation, which in turn causes phosphorylation of Ser32 and Ser36 sites of $\mathrm{IkB} \alpha$ (Hayden and Ghosh, 2004). Phosphorylated IkB $\alpha$ is then degraded to p50/p65 for nuclear translocation (Hayden and Ghosh, 2004; Oeckinghaus and Ghosh, 2009). Translocated $\mathrm{NF}-\kappa \mathrm{B}$ induces expression of anti-apoptotic genes, such as Bcl$\mathrm{Xl}$ and $\mathrm{Bcl}-2$, leading to enhance survival while avoiding apoptosis of most cells (Ward et al., 2004; Namba et al., 2007; Nguyen et al., 2014). Multiple chemotherapeutics play an anticancer role by blocking this pathway (Zhang K. et al., 2019). In OVCAR-3 cells, in addition to significantly affect the mitochondrial pathway, Polygala tenuifolia Willd. polysaccharide (PTP) treatment could reduce the expression of $\mathrm{NF}-\kappa \mathrm{B}$, thereby triggering cell death signals in the programmed external pathway of tumor cells (Zhang et al., 2015). A similar mechanism was found in Hordeum vulgare $L$. polysaccharide (BP-1) to stimulate human colon cancer cells (HT-29). BP-1 could inhibit the transfer of NF- $\kappa B$ from the cytoplasm to the nucleus. This further affected apoptosis-related proteins, such as $\mathrm{Bcl}-2$, promoted the release of cytochrome $\mathrm{c}$ from mitochondria to the cytoplasm, and the activation of Caspase- 8 and Caspase- 9 to induce apoptosis (Cheng et al., 2016). Furthermore, Portulaca oleracea L. Polysaccharide (POL-P3b) could inhibit the expression of TLR4, MyD88, TRAF6, AP-1, and NF- $\mathrm{B}$ 


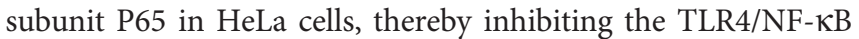
pathway to induce Cervical cancer cells apoptosis (Zhao R. et al., 2017).

\section{Other Pathways}

Apoptosis is regulated by multiple targets and multiple pathways. In addition to the above mechanisms, plant polysaccharides can affect many other pathways to induce apoptosis. CREB is a major inductor of apoptosis activation, ASP could regulate the activation of $\mathrm{Bcl}-2$ through $\mathrm{CREB}$ signaling pathway to induce apoptosis and to produce a profound antitumor effect on T47D cells (Zhou et al., 2015). Abnormal activation of STAT3 is necessary for the survival of many human cancer cells, and inhibition of JAK2/STAT3 pathway by inhibitors or siRNAs can reduce cell survival and induce apoptosis (Shu et al., 2011). Shen et al. reported that Pumpkin polysaccharide (PPPF)-induced apoptosis in HepG2 cells may be mediated by the JAK2/ STAT3 signal transduction pathway (Shen et al., 2017). The expression, abundance, and activity of ER $\alpha$ were increased in most of breast cancers (Lundqvist et al., 2018). Sparganium stoloniferum (Buch.-Ham. ex Graebn.) Buch.-Ham. ex Juz. polysaccharide (SpaTA) could regulate the expression of ER $\alpha$ and activate its genomic functions by inducing the downstream estrogen pathway, and then further activated the Caspase cascade to exert apoptosis in an ER $\alpha$-dependent manner ( $\mathrm{Wu} \mathrm{Y}$. et al., 2017). Twist and its downstream AKR1C2 and NF1 genes can induce tumor progression, cell proliferation and tumorigenesis in HGC-27 cells. It has been found that PGP2a-induced gastric cancer HGC-27 cell apoptosis may be mediated by regulating the expression of Twist and its downstream genes (Li C. et al., 2014). G2/M phase arrest is closely related to early cell apoptosis. SPS induced lung cancer cells cycle arrest in the G2/M phase by reducing the expression of $\mathrm{CDC} 25 \mathrm{~B}$ and cyclin $\mathrm{B} 1$ (Li J. Y. et al., 2016).

\section{THE ABSORPTION OF PLANT POLYSACCHARIDES IN VIVO}

Drugs must first enter the body in order to work, so the absorption pathways and bioavailability of plant polysaccharides should be discussed. Studies have found that polysaccharides may be absorbed by the intestine after oral administration. As early as 2014, Liao established a Caco-2 cell transport model and found that both Gastrodia polysaccharide and Grifola frondosa (Dicks). Gray polysaccharides could successfully transmembrane, and the polysaccharides remained basically unchanged without degradation. Further studies on its absorption pathway revealed that the uptake of two polysaccharides in the small intestinal cells is the clathrin heavy chain endocytosis pathway (Liao, 2014). A similar study was discovered in 2017. After oral administration of ASP, ASP could be absorbed through the large cell-drinking route and clathrin and small concave (or lipid raft) -related endocytosis, and enter the blood circulation to exert effect (Wang et al., 2017). While the absorption of Gynochthodes officinalis (F.C.How) Razafim. \&
B.Bremer (syn. Morinda officinalis F.C.How) polysaccharide in the small intestine was found to be accomplished through passive diffusion (Chen et al., 2012). However, in general, the oral absorption rate of polysaccharides is negatively affected by its molecular weight, and the bioavailability of most plant polysaccharides is low (Yi et al., 2014). To improve the bioavailability and pharmacological effects of bioactive polysaccharides, various particle delivery systems have been developed, such as liposomes, nanovesicles, nanoemulsions, and micelles (Li et al., 2017b; Ruttala et al., 2017). Morus alba L. (Fructus Mori) polysaccharides (MFP) were spheroidized by anti-solvent precipitation method. The oral bioavailability of MFP and its particles (MFP-NP1, MFP-NP2, MFP-NP3 and the size of their spheroidization is MFP-NP1> MFP-NP2> MFPNP3) were evaluated by measuring the polysaccharide content in the plasma of mice. After $4 \mathrm{~h}$ of administration, the concentrations of MFP, MFP-NP1, MFP-NP2 and MFP-NP3 were $0.28,0.58,0.69$, and $0.97 \mu \mathrm{g} / \mathrm{ml}$, respectively. The relative bioavailability of MFP- NP1, MFP- NP2, and MFP- NP3 are 4.81, 6.33 , and 8.54 times, respectively, which are inversely related to their size (Chen and $\mathrm{Fu}, 2019$ ). In addition, encapsulation of nanoencapsulated polysaccharides extracted from Taiwanofungus camphoratus (M. Zang \& C.H. Su) Sheng H. Wu, Z.H. Yu, Y.C. Dai \& C.H. Su (syn. Antrodia camphorata (M. Zang \& C.H. Su) Sheng H. Wu, Ryvarden \& T.T. Chang), enhanced its biological activity (Chang et al., 2015). The encapsulated nanoemulsion of Anthophycus longifolius (Turner) Kützing (syn. Sargassum longifolium (Turner) C.Agardh) polysaccharide was pretreated and sustainedly released in vitro, indicating an increase in its bioavailability (Shofia et al., 2018). Ophiopogon japonicus (Thunb.) Ker Gawl. polysaccharides (ROPs)-loaded liposomes showed more efficient and stronger antigen-specific immune responses than free ROPs (Fan et al., 2016). Interestingly, recent studies have shown that certain polysaccharides can inhibit tumors in the host. However, they cannot rely on absorption into the body to work due to their large molecular weight, but directly affect the gut microbiota. The gut microbiota has a symbiotic relationship with the host, plays a decisive role in host nutrition, immunity and metabolism, and it can regulate the effect of cancer treatment (Chen D. et al., 2019). For example, in a colorectal cancer mouse model, licorice and jujube polysaccharides positively regulated the intestinal microbial flora and affected certain metabolic pathways that are beneficial to the health of the host to exert antitumor effects (Zhang X. et al., 2018; Ji et al., 2020). After oral administration Dendrobium officinale Kimura \& Migo (syn. Dendrobium huoshanense Kimura \& Migo) polysaccharide, it could significantly change the physiological state of the intestine, enhance the function of the intestinal physiology, biochemistry and the intestinal immunological barrier, and regulate the composition of microbial population and microbial metabolism (Xie S. et al., 2019). In a study in 2019, D. officinale polysaccharide (DOP) was shown to have anti-breast cancer effects. But it was indigestible and non-absorbable, and eventually it was discovered that DOP exerted the effect by regulating the components of the intestinal flora related to anti-breast cancer (Li L. et al., 2019). 


\section{THE TOXICITY ANALYSIS OF PLANT POLYSACCHARIDES}

In the clinic, the treatment of cancer is a long-term process, so the study of drug toxicity is also necessary. The application of most plant polysaccharides to cancer shows no toxicity. Lung cancer was treated with SDP for 15 days, and all nude mice were observed to show no signs of death or toxicity. Further research found that SDP could induce A549 cells apoptosis, but had no significant effect on normal human fibroblasts WI38 (Lin et al., 2019). In addition, no mice died and no significant reduction in body weight was observed during 3 weeks of CAs-treated liver cancer, indicating that CAs have no significant toxicity to host animals (Zhao Y. et al., 2017). In one study, licorice polysaccharides have been shown to increase immune organ weight and index, activate immune cells, thereby inhibiting tumor growth, and had no toxic manifestations in CT 26 tumor-bearing mice (Ayeka et al., 2017). Chen et al. carried out a toxicity study of YLSP in male and female rats, and found that a single oral administration of $24 \mathrm{~g} / \mathrm{kg}$ YLSP in the acute toxicity study did not cause toxicological symptoms or cause death. Also, in the chronic toxicity study, intragastric administration was performed for 26 weeks, followed by a 3week recovery period, and no death or significant clinical symptoms were observed. In addition, no toxicity was found in measurements of body weight, food consumption, hematology, clinical biochemistry, organ weight (Chen et al., 2016). Male and female rats were given tamarind polysaccharide for 28 days without death and no pathological findings (Heimbach et al., 2013). In the toxicity test of Codonopsis pilosula (Franch.) Nannf. polysaccharide oral solution administered to rats, there were no significant changes in the general condition, behavior, hematology, blood biochemistry, and main organ pathology of rats in each group after 4 weeks of administration and two weeks after withdrawal (Hou et al., 2016). APS had no obvious toxicity in 30-day long-term toxicity test in rats. The growth and development of rats, viscera-body ratio, and other indicators did not show significant effects, and gross anatomy and histological observation showed no abnormal pathological changes (Liu and Xiao, 2011). In addition, plant polysaccharides from Aster tataricus, Panax ginseng, Angelica sinensis, Aralia elata (Miq). Seem., pumpkin and most others have no obvious toxic and side effects on normal cells or nude mice during the treatment process (Du et al., 2014; Li C. et al., 2014; Zhou et al., 2015; Liu et al., 2016b; Sun et al., 2016; Shen et al., 2017).

\section{CONCLUSIONS AND PERSPECTIVES}

Multi-component plants and their extracts are often used to successfully treat various diseases, including suppressing cancer. Polysaccharides are active ingredients widely present in a large number of plants, and they can induce apoptosis through multiple targets and multiple pathways in the treatment of cancer. Therefore, in this review, we evaluated related articles that revealed how fifty plant polysaccharides can induce apoptosis and play a role in improving cancer cells in a multi-target, multi- path way. Mitochondrial-mediated pathway, death receptor pathway, and their upstream signal transduction such as MAPK pathway, PI3K/AKT pathway and NF- $\kappa B$ pathway are important pathways that affect apoptosis. As shown in Figures 1-3, plant polysaccharides can effectively play a pro-apoptotic effect and effectively improve cancer through these pathways. However, different plant polysaccharides can perform different apoptotic pathways, and different plant polysaccharides have different effects and mechanisms for different types of cancer, as shown in Table 1. And the high cancer cell apoptosis rate, cancer inhibition rate, and low cancer cell vitality reflect the superior anti-cancer effect of plant polysaccharides. The activity of polysaccharides is expressed in different units, which is difficult to compare with traditional anticancer drugs. However, at normal dosages, some plant polysaccharides had the same strength in inhibiting cancer as radiotherapy and chemotherapy. In addition, polysaccharide activity is closely related to structural characteristics, but plant polysaccharides are mostly mixtures in nature with complex structures and various types. Isolation, purification and structure determination of polysaccharides are difficult. Therefore, as shown in Table 1, the structural characteristics of most of the reported active polysaccharides are still unclear, and structure-activity relationships cannot be studied in detail. Nevertheless, the structural diversity and activity of polysaccharides depend to a large extent on their plant source. We have concluded that most of polysaccharides that induce apoptosis and produce anticancer activity come from these plants which are both functional foods and medical drugs, this is what we often call drug and food homology. In daily life, these plants are often widely used to supplement nutrition, and no serious physical injuries have been found. Therefore, in the research of anticancer drugs, we can pay close attention to these plants or their components that are homologous to medicinal and food products, which may have both strong biological activity and excellent safety.

In addition, we found that after oral administration some plant polysaccharides can be absorbed in the small intestine by passive diffusion, endocytosis, and so on. However, due to the large molecular weight of most polysaccharides, their bioavailability is still generally low. Using nanometer and liposome for encapsulation was found to improve their bioavailability and bioactivity. Subsequently, the effect of plant polysaccharides on the intestinal microflora provides us with new ideas. This can reveal why plant polysaccharides are not well absorbed but can inhibit tumor growth in vivo. So, in the subsequent research on the anticancer mechanism of plant polysaccharides, we can take influencing the gut microbiota as an important direction.

The ultimate purpose of the drug is to serve the clinic. Studies have shown that most plant polysaccharides have obvious anticancer effects and are non-toxic to normal organisms, ensuring clinical effectiveness and safety. However, the existing clinical research on plant polysaccharides is limited, and most polysaccharides have not been studied in human-specific clinical trials. One possible reason is that most plant polysaccharides are currently focused on anti-cancer 
mechanism research, but no overall animal experiments have been conducted to determine the efficacy of polysaccharides in vivo. Consequently, it is hoped that in the future research, the anticancer effect of plant polysaccharides in vivo can be comprehensively researched and entered into the clinical research stage as soon as possible.

\section{AUTHOR CONTRIBUTIONS}

Q-WH is the corresponding author on the study. Q-XG and JW are first authors and responsible for collecting materials and writing the paper. JH, G-HL, H-JX, and C-YP helped in organizing the information and edited the article pictures. All authors read and approved the final manuscript.

\section{REFERENCES}

Agool, A., Slart, R. H., Thorp, K. K., Glaudemans, A. W., Cobben, D. C., Been, L. B., et al. (2011). Effect of radiotherapy and chemotherapy on bone marrow activity: a 18F-FLT-PET study. Nucl. Med. Commun. 32 (1), 17-22. doi: 10.1097/ mnm.0b013e328340798c

Arlt, A., Müerköster, S. S., and Schäfer, H. (2013). Targeting apoptosis pathways in pancreatic cancer. Cancer Lett. 332 (2), 346-358. doi: 10.1016/ j.canlet.2010.10.015

Ayeka, P. A., Bian, Y., Githaiga, P. M., and Zhao, Y. (2017). The immunomodulatory activities of licorice polysaccharides (Glycyrrhiza uralensis Fisch.) in CT 26 tumor-bearing mice. BMC Complement. Altern. Med. 17 (1), 536. doi: 10.1186/s12906-017-2030-7

Bishayee, A., and Sethi, G. (2016). Bioactive natural products in cancer prevention and therapy: Progress and promise. Semin. Cancer Biol., 1-3. doi: 10.1016/ j.semcancer.2016.08.006

Cagnol, S., and Chambard, J. C. (2010). ERK and cell death: mechanisms of ERKinduced cell death-apoptosis, autophagy and senescence. FEBS J. 277 (1), 2-21. doi: $10.1111 / j .1742-4658.2009 .07366 . x$

Chang, F., Lee, J. T., Navolanic, P. M., Steelman, L. S., Shelton, J. G., Blalock, W. L., et al. (2003). Involvement of PI3K/Akt pathway in cell cycle progression, apoptosis, and neoplastic transformation: a target for cancer chemotherapy. Leukemia 17 (3), 590-603. doi: 10.1038/sj.leu.2402824

Chang, J. S., Kuo, H. P., Chang, K. L., and Kong, Z. L. (2015). Apoptosis of Hepatocellular Carcinoma Cells Induced by Nanoencapsulated Polysaccharides Extracted from Antrodia Camphorata. PloS One 10 (9), e0136782. doi: 10.1371/journal.pone.0136782

Cheki, M., Yahyapour, R., Farhood, B., Rezaeyan, A., Shabeeb, D., Amini, P., et al. (2018). COX-2 in Radiotherapy: A Potential Target for Radioprotection and Radiosensitization. Curr. Mol. Pharmacol. 11 (3), 173-183. doi: 10.2174/ 1874467211666180219102520

Chen, C., and Fu, X. (2019). Spheroidization on Fructus Mori polysaccharides to enhance bioavailability and bioactivity by anti-solvent precipitation method. Food Chem. 300, 125245. doi: 10.1016/j.foodchem.2019.125245

Chen, D., Chen, Z., Lin, L., Chen, K., Zhang, P., Liang, X., et al. (2012). In-vivo Intestinal Absorption Mechanism Study for Polysaccharides of Morindae ficinalis. China New Drugs Clin. Pharmacol. 23 (2), 61-64. doi: CNKI:SUN: ZYXY.0.2012-01-020

Chen, J., Jin, X., Chen, J., and Liu, C. (2013a). Glycyrrhiza polysaccharide induces apoptosis and inhibits proliferation of human hepatocellular carcinoma cells by blocking PI3K/AKT signal pathway. Tumour Biol. 34 (3), 1381-1389. doi: 10.1007/s13277-013-0746-7

Chen, J., Yao, D., Yuan, H., Zhang, S., Tian, J., Guo, W., et al. (2013b). Dipsacus asperoides polysaccharide induces apoptosis in osteosarcoma cells by modulating the PI3K/Akt pathway. Carbohydr. Polym. 95 (2), 780-784. doi: 10.1016/j.carbpol.2013.03.009

\section{FUNDING}

This work is financially supported by the Key R \& D projects (Code:2018SZ0077) and the applied basic research project (Code:2018JY0032), both of Sichuan Science and Technology Department, by the Chengdu Science and Technology Bureau Technology R \& D Project (Code: 2015-HM01-00401-SF)

\section{ACKNOWLEDGMENTS}

We are indebted to our alma mater, Chengdu University of Traditional Chinese Medicine for provided convenience in the collection of documents. Thanks for all the help from everyone in our lab.

Chen, C., Nong, Z., Meng, M., Wen, Q., Lin, X., Qin, F., et al. (2016). Toxicological evaluation of Yulangsan polysaccharide in Wistar rats: A 26-week oral gavage study. Environ. Toxicol. Pharmacol. 41, 1-7. doi: 10.1016/j.etap.2015.10.011

Chen, D., Sun, S., Cai, D., and Kong, G. (2017). Induction of mitochondrialdependent apoptosis in T24 cells by a selenium (Se)-containing polysaccharide from Ginkgo biloba L. leaves. Int. J. Biol. Macromol. 101, 126-130. doi: 10.1016/j.ijbiomac.2017.03.033

Chen, C., Ma, Y., Li, Y.-D., and Zhang, X.-C. (2019). Influence of LBP alone or Combined with TRAIL on Apoptosis of MLL Rearranged Leukemic Cells. J. Exp. Hematol. 27 (4), 1104-1110. doi: 10.19746/j.cnki.issn1009-2137. 2019.04.018

Chen, D., Wu, J., Jin, D., Wang, B., and Cao, H. (2019). Fecal microbiota transplantation in cancer management: Current status and perspectives. Int. J. Cancer 145 (8), 2021-2031. doi: 10.1002/ijc.32003

Cheng, D., Zhang, X., Meng, M., Han, L., Li, C., Hou, L., et al. (2016). Inhibitory effect on HT-29 colon cancer cells of a water-soluble polysaccharide obtained from highland barley. Int. J. Biol. Macromol. 92, 88-95. doi: 10.1016/ j.ijbiomac.2016.06.099

Cho, H. D., Lee, J. H., Moon, K. D., Park, K. H., Lee, M. K., and Seo, K. I. (2018). Auriculasin-induced ROS causes prostate cancer cell death via induction of apoptosis. Food Chem. Toxicol. 111, 660-669. doi: 10.1016/j.fct.2017.12.007

Chu, Y., Fang, Y., Chi, J., Li, J., Zhang, D., Zou, Y., et al. (2018). Astragalus polysaccharides decrease proliferation, migration, and invasion but increase apoptosis of human osteosarcoma cells by up-regulation of microRNA-133a. Braz. J. Med. Biol. Res. 51 (12), e7665. doi: 10.1590/1414-431x20187665

Deng, Q., Huang, C. M., Chen, N., Li, L., Wang, X. D., Zhang, W., et al. (2012). Chemotherapy and radiotherapy downregulate the activity and expression of DNA methyltransferase and enhance Bcl-2/E1B-19-kDa interacting protein-3induced apoptosis in human colorectal cancer cells. Chemotherapy 58 (6), 445453. doi: $10.1159 / 000345916$

Dhanasekaran, D. N., and Reddy, E. P. (2008). JNK signaling in apoptosis. Oncogene 27 (48), 6245-6251. doi: 10.1038/onc.2008.301

Dong, K., Lei, Q., Guo, R., Wu, X., Zhang, Y., Cui, N., et al. (2019). Regulating intracellular ROS signal by a dual $\mathrm{pH} /$ reducing-responsive nanogels system promotes tumor cell apoptosis. Int. J. Nanomed. 14, 5713-5728. doi: 10.2147/ ijn.s208089

Du, L., Mei, H. F., Yin, X., and Xing, Y. Q. (2014). Delayed growth of glioma by a polysaccharide from Aster tataricus involve upregulation of $\mathrm{Bax} / \mathrm{Bcl}-2$ ratio, activation of caspase-3/8/9, and downregulation of the Akt. Tumour Biol. 35 (3), 1819-1825. doi: 10.1007/s13277-013-1243-8

Duan, H., Wang, B., and Zhang, Y. (2014). Anti-tumor Effects and Mechanism of Rhizoma Polygonati Polysaccharide on H 22 Tumor Bearing Mice. Tradit. Chin. Drug Res. \& Clin. Pharmacol. 25 (1), 5-7. doi: 10.3969/j.issn.10039783.2014.01.002

Fan, Y., Ma, X., Ma, L., Zhang, J., Zhang, W., and Song, X. (2016). Antioxidative and immunological activities of ophiopogon polysaccharide liposome from the 
root of Ophiopogon japonicus. Carbohydr. Polym. 135, 110-120. doi: 10.1016/ j.carbpol.2015.08.089

Feng, G., Wang, X., You, C., Cheng, X., Han, Z., Zong, L., et al. (2013). Antiproliferative potential of Artemisia capillaris polysaccharide against human nasopharyngeal carcinoma cells. Carbohydr. Polym. 92 (2), 10401045. doi: 10.1016/j.carbpol.2012.10.024

Franke, T. F., Hornik, C. P., Segev, L., Shostak, G. A., and Sugimoto, C. (2003). PI3K/Akt and apoptosis: size matters. Oncogene 22 (56), 8983-8998. doi: $10.1038 /$ sj.onc. 1207115

Guo, L., Bai, S. P., Zhao, L., and Wang, X. H. (2012). Astragalus polysaccharide injection integrated with vinorelbine and cisplatin for patients with advanced non-small cell lung cancer: effects on quality of life and survival. Med. Oncol. 29 (3), 1656-1662. doi: 10.1007/s12032-011-0068-9

Hayden, M. S., and Ghosh, S. (2004). Signaling to NF-kappaB. Genes Dev. 18 (18), 2195-2224. doi: 10.1101/gad.1228704

Heimbach, J. T., Egawa, H., Marone, P. A., Bauter, M. R., and Kennepohl, E. (2013). Tamarind seed polysaccharide: a 28-day dietary study in SpragueDawley rats. Int. J. Toxicol. 32 (3), 198-208. doi: 10.1177/1091581813484069

Heinicke, U., Haydn, T., Kehr, S., Vogler, M., and Fulda, S. (2018). BCL-2 selective inhibitor ABT-199 primes rhabdomyosarcoma cells to histone deacetylase inhibitor-induced apoptosis. Oncogene 37 (39), 5325-5339. doi: 10.1038/s41388018-0212-5

Hou, L., Han, L., Gong, Z., and Yan, Y. (2016). Long-term Toxicity of Codonopsis Polysaccharide Oral Liquid in Rats. Chin. J. Veterin. Drugs 50 (12), 40-44.

Hsieh, C. H., Lin, C. Y., Hsu, C. L., Fan, K. H., Huang, S. F., Liao, C. T., et al. (2020). Incorporation of Astragalus polysaccharides injection during concurrent chemoradiotherapy in advanced pharyngeal or laryngeal squamous cell carcinoma: preliminary experience of a phase II double-blind, randomized trial. J. Cancer Res. Clin. Oncol. 146 (1), 33-41. doi: 10.1007/s00432-019-03033-8

Huang, X., Zou, L., Yu, X., Chen, M., Guo, R., Cai, H., et al. (2015). Salidroside attenuates chronic hypoxia-induced pulmonary hypertension via adenosine A2a receptor related mitochondria-dependent apoptosis pathway. J. Mol. Cell. Cardiol. 82, 153-166. doi: 10.1016/j.yjmcc.2015.03.005

Huang, W. H., Liao, W. R., and Sun, R. X. (2016). Astragalus polysaccharide induces the apoptosis of human hepatocellular carcinoma cells by decreasing the expression of Notch1. Int. J. Mol. Med. 38 (2), 551-557. doi: 10.3892/ ijmm. 2016.2632

Huang, T. C., Chiu, P. R., Chang, W. T., Hsieh, B. S., Huang, Y. C., Cheng, H. L., et al. (2018). Epirubicin induces apoptosis in osteoblasts through deathreceptor and mitochondrial pathways. Apoptosis 23, 226-236. doi: 10.1007/ s10495-018-1450-2

Iurlaro, R., and Muñoz-Pinedo, C. (2016). Cell death induced by endoplasmic reticulum stress. FEBS J. 283 (14), 2640-2652. doi: 10.1111/febs.13598

Ji, X., Hou, C., Gao, Y., Xue, Y., Yan, Y., and Guo, X. (2020). Metagenomic analysis of gut microbiota modulatory effects of jujube (Ziziphus jujuba Mill.) polysaccharides in a colorectal cancer mouse model. Food Funct. 11 (1), 163-173. doi: 10.1039/c9fo02171j

Jiang, W., Fu, Y., Yang, F., Yang, Y., Liu, T., Zheng, W., et al. (2014). Gracilaria lemaneiformis polysaccharide as integrin-targeting surface decorator of selenium nanoparticles to achieve enhanced anticancer efficacy. ACS Appl. Mater. Interf. 6 (16), 13738-13748. doi: 10.1021/am5031962

Jiao, R., Liu, Y., Gao, H., Xiao, J., and So, K. F. (2016). The Anti-Oxidant and Antitumor Properties of Plant Polysaccharides. Am. J. Chin. Med. 44 (3), $463-$ 488. doi: $10.1142 / \mathrm{s} 0192415 \times 16500269$

Jin, L. Q., Zheng, Z. J., Peng, Y., Li, W. X., Chen, X. M., and Lu, J. X. (2007). Opposite effects on tumor growth depending on dose of Achyranthes bidentata polysaccharides in C57BL/6 mice. Int. Immunopharmacol. 7 (5), 568-577. doi: 10.1016/j.intimp.2006.12.009

Kang, Y., Wang, Z. J., Xie, D., Sun, X., Yang, W., Zhao, X., et al. (2017). Characterization and Potential Antitumor Activity of Polysaccharide from Gracilariopsis lemaneiformis. Mar. Drugs 15 (4), 100-114. doi: 10.3390/ md15040100

Kaufmann, T., Strasser, A., and Jost, P. J. (2012). Fas death receptor signalling: roles of Bid and XIAP. Cell Death Differ. 19 (1), 42-50. doi: 10.1038/cdd.2011.121

Khan, F. A., Pandupuspitasari, N. S., Chun-Jie, H., Ao, Z., Jamal, M., Zohaib, A., et al. (2016). CRISPR/Cas9 therapeutics: a cure for cancer and other genetic diseases. Oncotarget 7 (32), 52541-52552. doi: 10.18632/oncotarget.9646
Khan, T., Date, A., Chawda, H., and Patel, K. (2019). Polysaccharides as potential anticancer agents-A review of their progress. Carbohydr. Polym. 210, 412-428. doi: 10.1016/j.carbpol.2019.01.064

Kopeina, G. S., Prokhorova, E. A., Lavrik, I. N., and Zhivotovsky, B. (2018). Alterations in the nucleocytoplasmic transport in apoptosis: Caspases lead the way. Cell Prolif. 51 (5), e12467. doi: 10.1111/cpr.12467

Kwon, M. J., and Nam, T. J. (2007). A polysaccharide of the marine alga Capsosiphon fulvescens induces apoptosis in AGS gastric cancer cells via an IGF-IR-mediated PI3K/Akt pathway. Cell Biol. Int. 31 (8), 768-775. doi: 10.1016/j.cellbi.2007.01.010

Li, Y., and Sun, S.-F. (2019). Effect of Cornus officinalis polysaccharide on proliferation and apoptosis of hepatocellular carcinoma HepG2 cells by up-regulating Klotho expression and inhibiting PI3K/ AKT pathway. Drugs Clin. 34 (10), 2887-2893. doi: 10.7501/j.issn.1674-5515.2019. 10.002

Li, C., Tian, Z. N., Cai, J. P., Chen, K. X., Zhang, B., Feng, M. Y., et al. (2014). Panax ginseng polysaccharide induces apoptosis by targeting Twist/AKR1C2/NF-1 pathway in human gastric cancer. Carbohydr. Polym. 102, 103-109. doi: 10.1016/j.carbpol.2013.11.016

Li, J., Zhang, F., and Wang, S. (2014). A polysaccharide from pomegranate peels induces the apoptosis of human osteosarcoma cells via the mitochondrial apoptotic pathway. Tumour Biol. 35 (8), 7475-7482. doi: 10.1007/s13277-0141983-0

Li, X., Liu, F., Li, Z., Ye, N., Huang, C., and Yuan, X. (2014). Atractylodes macrocephala polysaccharides induces mitochondrial-mediated apoptosis in glioma C6 cells. Int. J. Biol. Macromol. 66, 108-112. doi: 10.1016/ j.ijbiomac.2014.02.019

Li, J. Y., Yu, J., Du, X. S., Zhang, H. M., Wang, B., Guo, H., et al. (2016). Safflower polysaccharide induces NSCLC cell apoptosis by inhibition of the Akt pathway. Oncol. Rep. 36 (1), 147-154. doi: 10.3892/or.2016.4784

Li, Y., Li, D., Chen, J., and Wang, S. (2016). A polysaccharide from Pinellia ternata inhibits cell proliferation and metastasis in human cholangiocarcinoma cells by targeting of Cdc42 and $67 \mathrm{kDa}$ Laminin Receptor (LR). Int. J. Biol. Macromol. 93 (Pt A), 520-525. doi: 10.1016/j.ijbiomac.2016.08.069

Li, W., Yu, K. N., Ma, J., Shen, J., Cheng, C., Zhou, F., et al. (2017a). Non-thermal plasma induces mitochondria-mediated apoptotic signaling pathway via ROS generation in HeLa cells. Arch. Biochem. Biophys. 633, 68-77. doi: 10.1016/ j.abb.2017.09.005

Li, X., Wang, L., and Wang, B. (2017b). Optimization of encapsulation efficiency and average particle size of Hohenbuehelia serotina polysaccharides nanoemulsions using response surface methodology. Food Chem. 229, 479486. doi: 10.1016/j.foodchem.2017.02.051

Li, C., Hong, L., Liu, C., Min, J., Hu, M., and Guo, W. (2018). Astragalus polysaccharides increase the sensitivity of SKOV3 cells to cisplatin. Arch. Gynecol. Obstet. 297 (2), 381-386. doi: 10.1007/s00404-017-4580-9

Li, Y., Wang, K., Yin, Y., Li, Y., and Li, S. (2018). Relationships between family resilience, breast cancer survivors' individual resilience, and caregiver burden: A cross-sectional study. Int. J. Nurs. Stud. 88, 79-84. doi: 10.1016/ j.ijnurstu.2018.08.011

Li, L., Yao, H., Li, X., Zhang, Q., Wu, X., Wong, T., et al. (2019). Destiny of Dendrobium officinale Polysaccharide after Oral Administration: Indigestible and Nonabsorbing, Ends in Modulating Gut Microbiota. J. Agric. Food Chem. 67 (21), 5968-5977. doi: 10.1021/acs.jafc.9b01489

Li, W., Song, K., Wang, S., Zhang, C., Zhuang, M., Wang, Y., et al. (2019). Antitumor potential of astragalus polysaccharides on breast cancer cell line mediated by macrophage activation. Mater. Sci. Eng. C. Mater. Biol. Appl. 98, 685-695. doi: 10.1016/j.msec.2019.01.025

Liang, M., Liu, J., Ji, H., Chen, M., Zhao, Y., Li, S., et al. (2015). A Aconitum coreanum polysaccharide fraction induces apoptosis of hepatocellular carcinoma (HCC) cells via pituitary tumor transforming gene 1 (PTTG1)mediated suppression of the P13K/Akt and activation of p38 MAPK signaling pathway and displays antitumor activity in vivo. Tumour Biol. 36 (9), 70857091. doi: 10.1007/s13277-015-3420-4

Liang, Y., Xu, W., Liu, S., Chi, J., Zhang, J., Sui, A., et al. (2018). N-AcetylGlucosamine Sensitizes Non-Small Cell Lung Cancer Cells to TRAIL-Induced Apoptosis by Activating Death Receptor 5. Cell. Physiol. Biochem. 45 (5), $2054-$ 2070. doi: $10.1159 / 000488042$ 
Liao, W. (2014). Study on the Activity of Traditional Chinese Medicine Polysaccharide and Its Oral Absorption Mechanism (Chinese Academy of Sciences University)

Lin, L., Cheng, K., Xie, Z., Chen, C., Chen, L., Huang, Y., et al. (2019). Purification and characterization a polysaccharide from Hedyotis diffusa and its apoptosis inducing activity toward human lung cancer cell line A549. Int. J. Biol. Macromol. 122, 64-71. doi: 10.1016/j.ijbiomac.2018.10.077

Liu, S., and Xiao, Y. (2011). Study on Toxicity of Astragalus Polysaccharide. Northern Med. 6 (2), 20-21.

Liu, S. J., Qu, H. M., and Ren, Y. P. (2014). SCP, a polysaccharide from Schisandra chinensis, induces apoptosis in human renal cell carcinoma Caki-1 cells through mitochondrial-dependent pathway via inhibition of ERK activation. Tumour Biol. 35 (6), 5369-5374. doi: 10.1007/s13277-014-1699-1

Liu, G., Kuang, S., Wu, S., Jin, W., and Sun, C. (2016a). A novel polysaccharide from Sargassum integerrimum induces apoptosis in A549 cells and prevents angiogensis in vitro and in vivo. Sci. Rep. 6, 26722. doi: 10.1038/srep26722

Liu, G., Sheng, Y., Zhang, M., and Sun, D. (2016b). A polysaccharide from the leaves of Aralia elata induces apoptosis in U-2 OS cells via mitochondrialdependent pathway. Int. J. Biol. Macromol. 93 (Pt A), 418-425. doi: 10.1016/ j.ijbiomac.2016.08.067

Liu, Z., Huang, P., Law, S., Tian, H., Leung, W., and Xu, C. (2018). Preventive Effect of Curcumin Against Chemotherapy-Induced Side-Effects. Front. Pharmacol. 9, 1374. doi: 10.3389/fphar.2018.01374

Liu, J. S., Huo, C. Y., Cao, H. H., Fan, C. L., Hu, J. Y., Deng, L. J., et al. (2019). Aloperine induces apoptosis and G2/M cell cycle arrest in hepatocellular carcinoma cells through the PI3K/Akt signaling pathway. Phytomedicine 61, 152843. doi: 10.1016/j.phymed.2019.152843

Liu, Y. (2008). Clinical observation of ginseng polysaccharide injection combined with chemotherapy in the treatment of advanced malignant tumors. China Clin. Prac. Med. 2 (12), 83-84.

López-Gómez, M., Malmierca, E., de Górgolas, M., and Casado, E. (2013). Cancer in developing countries: the next most preventable pandemic. The global problem of cancer. Crit. Rev. Oncol. Hematol. 88 (1), 117-122. doi: 10.1016/ j.critrevonc.2013.03.011

Ludwig-Galezowska, A. H., Flanagan, L., and Rehm, M. (2011). Apoptosis repressor with caspase recruitment domain, a multifunctional modulator of cell death. J. Cell. Mol. Med. 15 (5), 1044-1053. doi: 10.1111/j.15824934.2010.01221.x

Lundqvist, J., Kirkegaard, T., Laenkholm, A. V., Duun-Henriksen, A. K., Bak, M., Feldman, D., et al. (2018). Williams syndrome transcription factor (WSTF) acts as an activator of estrogen receptor signaling in breast cancer cells and the effect can be abrogated by 1 $\alpha, 25$-dihydroxyvitamin D. J. Steroid Biochem. 177, 171-178. doi: 10.1016/j.jsbmb.2017.06.003

Luo, Z., Zeng, H., Ye, Y., Liu, L., Li, S., Zhang, J., et al. (2015). Safflower polysaccharide inhibits the proliferation and metastasis of MCF-7 breast cancer cell. Mol. Med. Rep. 11 (6), 4611-4616. doi: 10.3892/mmr.2015.3310

Milisav, I., Poljšak, B., and Ribarič, S. (2017). Reduced risk of apoptosis: mechanisms of stress responses. Apoptosis 22 (2), 265-283. doi: 10.1007/ s10495-016-1317-3

Mortezaee, K., Najafi, M., Farhood, B., Ahmadi, A., Potes, Y., Shabeeb, D., et al. (2019a). Modulation of apoptosis by melatonin for improving cancer treatment efficiency: An updated review. Life Sci. 228, 228-241. doi: 10.1016/ j.lfs.2019.05.009

Mortezaee, K., Salehi, E., Mirtavoos-Mahyari, H., Motevaseli, E., Najafi, M., Farhood, B., et al. (2019b). Mechanisms of apoptosis modulation by curcumin: Implications for cancer therapy. J. Cell. Physiol. 234 (8), 1253712550. doi: $10.1002 /$ jcp. 28122

Murad, H., Hawat, M., Ekhtiar, A., AlJapawe, A., Abbas, A., Darwish, H., et al. (2016). Induction of G1-phase cell cycle arrest and apoptosis pathway in MDA-MB-231 human breast cancer cells by sulfated polysaccharide extracted from Laurencia papillosa. Cancer Cell Int. 16, 39. doi: 10.1186/s12935-016-0315-4

Namba, H., Saenko, V., and Yamashita, S. (2007). Nuclear factor-kB in thyroid carcinogenesis and progression: a novel therapeutic target for advanced thyroid cancer. Arq. Bras. Endocrinol. Metabol. 51 (5), 843-851. doi: 10.1590/s000427302007000500023

Nguyen, D. P., Li, J., Yadav, S. S., and Tewari, A. K. (2014). Recent insights into NF- $\mathrm{\kappa B}$ signalling pathways and the link between inflammation and prostate cancer. BJU Int. 114 (2), 168-176. doi: 10.1111/bju.12488
Oeckinghaus, A., and Ghosh, S. (2009). The NF-kappaB family of transcription factors and its regulation. Cold Spring Harb. Perspect. Biol. 1 (4), a000034. doi: 10.1101/cshperspect.a000034

Oun, R., Moussa, Y. E., and Wheate, N. J. (2018). The side effects of platinumbased chemotherapy drugs: a review for chemists. Dalton Trans. 47 (19), 66456653. doi: $10.1039 / \mathrm{c} 8 \mathrm{dt} 00838 \mathrm{~h}$

Panka, D. J., Atkins, M. B., and Mier, J. W. (2006). Targeting the mitogen-activated protein kinase pathway in the treatment of malignant melanoma. Clin. Cancer Res. 12, 2371-2375. doi: 10.1158/1078-0432.ccr-05-2539

Papa, S., Choy, P. M., and Bubici, C. (2019). The ERK and JNK pathways in the regulation of metabolic reprogramming. Oncogene 38 (13), 2223-2240. doi: 10.1038/s41388-018-0582-8

Petrache, I., Choi, M. E., Otterbein, L. E., Chin, B. Y., Mantell, L. L., Horowitz, S., et al. (1999). Mitogen-activated protein kinase pathway mediates hyperoxiainduced apoptosis in cultured macrophage cells. Am. J. Physiol. 277 (3), L589L595. doi: 10.1152/ajplung.1999.277.3.L589

Prenek, L., Boldizsár, F., Kugyelka, R., Ugor, E., Berta, G., Németh, P., et al. (2017). The regulation of the mitochondrial apoptotic pathway by glucocorticoid receptor in collaboration with $\mathrm{Bcl}-2$ family proteins in developing $\mathrm{T}$ cells. Apoptosis 22 (2), 239-253. doi: 10.1007/s10495-016-1320-8

Qin, Z., Lu, L., Yuan, G., Wu, G., Xue, S., and Gao, L. (2009). Clinical observation of astragalus polysaccharide for injection combined with GP regimen in the treatment of advanced non-small cell lung cancer. Chin. J. Tradit. Chin. Med. (3), 217-219. doi: CNKI:SUN:ZYHS.0.2009-03-095

Qin, N., Lu, S., Chen, N., Chen, C., Xie, Q., Wei, X., et al. (2019). Yulangsan polysaccharide inhibits $4 \mathrm{~T} 1$ breast cancer cell proliferation and induces apoptosis in vitro and in vivo. Int. J. Biol. Macromol. 121, 971-980. doi: 10.1016/j.ijbiomac.2018.10.082

Qu, H., Yang, W., and Li, J. (2018). Structural characterization of a polysaccharide from the flower buds of Tussilago farfara, and its effect on proliferation and apoptosis of A549 human non-small lung cancer cell line. Int. J. Biol. Macromol. 113, 849-858. doi: 10.1016/j.ijbiomac.2018.03.005

Rohlenova, K., Neuzil, J., and Rohlena, J. (2016). The role of Her2 and other oncogenes of the PI3K/AKT pathway in mitochondria. Biol. Chem. 397 (7), 607-615. doi: 10.1515/hsz-2016-0130

Rosell, R., and Karachaliou, N. (2015). Relationship between gene mutation and lung cancer metastasis. Cancer Metastasis Rev. 34 (2), 243-248. doi: 10.1007/ s10555-015-9557-1

Ruttala, H. B., Ramasamy, T., Gupta, B., Choi, H. G., Yong, C. S., and Kim, J. O. (2017). Multiple polysaccharide-drug complex-loaded liposomes: A unique strategy in drug loading and cancer targeting. Carbohydr. Polym. 173, 57-66. doi: 10.1016/j.carbpol.2017.05.062

Saad, A. M., Gad, M. M., Al-Husseini, M. J., AlKhayat, M. A., Rachid, A., Alfaar, A. S., et al. (2019). Suicidal death within a year of a cancer diagnosis: A population-based study. Cancer 125 (6), 972-979. doi: 10.1002/ cncr. 31876

Sadeghi, S., Davoodvandi, A., Pourhanifeh, M. H., Sharifi, N., ArefNezhad, R., Sahebnasagh, R., et al. (2019). Anti-cancer effects of cinnamon: Insights into its apoptosis effects. Eur. J. Med. Chem. 178, 131-140. doi: 10.1016/ j.ejmech.2019.05.067

Shen, H., and Laird, P. W. (2013). Interplay between the cancer genome and epigenome. Cell 153 (1), 38-55. doi: 10.1016/j.cell.2013.03.008

Shen, W., Guan, Y., Wang, J., Hu, Y., Tan, Q., Song, X., et al. (2016). A polysaccharide from pumpkin induces apoptosis of HepG2 cells by activation of mitochondrial pathway. Tumour Biol. 37 (4), 5239-5245. doi: 10.1007/s13277-015-4338-6

Shen, W., Chen, C., Guan, Y., Song, X., Jin, Y., Wang, J., et al. (2017). A pumpkin polysaccharide induces apoptosis by inhibiting the JAK2/STAT3 pathway in human hepatoma HepG2 cells. Int. J. Biol. Macromol. 104 (Pt A), 681-686. doi: 10.1016/j.ijbiomac.2017.06.078

Shofia, S. I., Jayakumar, K., Mukherjee, A., and Chandrasekaran, N. (2018). Efficiency of brown seaweed (Sargassum longifolium) polysaccharides encapsulated in nanoemulsion and nanostructured lipid carrier against colon cancer cell lines HCT 116. RSC Adv. 8 (29), 15973-15984. doi: 10.1039/ C8RA02616E

Shu, M., Zhou, Y., Zhu, W., Wu, S., Zheng, X., and Yan, G. (2011). Activation of a pro-survival pathway IL-6/JAK2/STAT3 contributes to glial fibrillary acidic protein induction during the cholera toxin-induced differentiation of C6 
malignant glioma cells. Mol. Oncol. 5 (3), 265-272. doi: 10.1016/ j.molonc.2011.03.003

Srivastava, S., Koay, E. J., Borowsky, A. D., De Marzo, A. M., Ghosh, S., Wagner, P. D., et al. (2019). Cancer overdiagnosis: a biological challenge and clinical dilemma. Nat. Rev. Cancer 19 (6), 349-358. doi: 10.1038/s41568-019-0142-8

Sun, F., Song, B., Wang, Y., and Jia, X. (2014). Clinical Study of Astragal Polysaccharides Combined With Iodine-125 in Treatment of Advanced Non-small Cell Lung Cancer. Chin. J. Exp. Tradit. Med. Formul. 20 (1), 189192. doi: $10.11653 /$ syfj2014010189

Sun, Q., Dong, M., Wang, Z., Wang, C., Sheng, D., Li, Z., et al. (2016). Seleniumenriched polysaccharides from Pyracantha fortuneana (Se-PFPs) inhibit the growth and invasive potential of ovarian cancer cells through inhibiting $\beta$ catenin signaling. Oncotarget 7 (19), 28369-28383. doi: 10.18632/ oncotarget.8619

Sun, P., Sun, D., and Wang, X. (2017). Effects of Scutellaria barbata polysaccharide on the proliferation, apoptosis and EMT of human colon cancer HT29 Cells. Carbohydr. Polym. 167, 90-96. doi: 10.1016/j.carbpol.2017.03.022

Sun, S., Zhang, C., Gao, J., Qin, Q., Zhang, Y., Zhu, H., et al. (2018). Benzoquinone induces ROS-dependent mitochondria-mediated apoptosis in HL-60 cells. Toxicol. Ind. Health 34 (4), 270-281. doi: 10.1177/0748233717750983

Sun, L. R., Zhou, W., Zhang, H. M., Guo, Q. S., Yang, W., Li, B. J., et al. (2019). Modulation of Multiple Signaling Pathways of the Plant-Derived Natural Products in Cancer. Front. Oncol. 9, 1153. doi: 10.3389/fonc.2019.01153

Surbone, A., and Halpern, M. T. (2016). Unequal cancer survivorship care: addressing cultural and sociodemographic disparities in the clinic. Support Care Cancer. 24 (12), 4831-4833. doi: 10.1007/s00520-016-3435-4

Thangam, R., Sathuvan, M., Poongodi, A., Suresh, V., Pazhanichamy, K., Sivasubramanian, S., et al. (2014). Activation of intrinsic apoptotic signaling pathway in cancer cells by Cymbopogon citratus polysaccharide fractions. Carbohydr. Polym. 107, 138-150. doi: 10.1016/j.carbpol.2014.02.039

Vaikundamoorthy, R., Krishnamoorthy, V., Vilwanathan, R., and Rajendran, R. (2018). Structural characterization and anticancer activity (MCF7 and MDAMB-231) of polysaccharides fractionated from brown seaweed Sargassum wightii. Int. J. Biol. Macromol. 111, 1229-1237. doi: 10.1016/j.ijbiomac.2018.01.125

Van Opdenbosch, N., and Lamkanfi, M. (2019). Caspases in Cell Death, Inflammation, and Disease. Immunity 50 (6), 1352-1364. doi: 10.1016/ j.immuni.2019.05.020

Veenstra, C. M., Regenbogen, S. E., Hawley, S. T., Griggs, J. J., Banerjee, M., Kato, I., et al. (2014). A composite measure of personal financial burden among patients with stage III colorectal cancer. Med. Care 52 (11), 957-962. doi: $10.1097 / \mathrm{mlr} .0000000000000241$

Wang, Z., Lu, C., Wu, C., Xu, M., Kou, X., Kong, D., et al. (2014). Polysaccharide of Boschniakia rossica induces apoptosis on laryngeal carcinoma Hep2 cells. Gene 536 (1), 203-206. doi: 10.1016/j.gene.2013.11.090

Wang, H., Li, J., Chi, H., Zhang, F., Zhu, X., Cai, J., et al. (2015). MicroRNA-181c targets Bcl-2 and regulates mitochondrial morphology in myocardial cells. J. Cell. Mol. Med. 19 (9), 2084-2097. doi: 10.1111/jcmm.12563

Wang, K., Cheng, F., Pan, X., Zhou, T., Liu, X., Zheng, Z., et al. (2017). Investigation of the transport and absorption of Angelica sinensis polysaccharide through gastrointestinal tract both in vitro and in vivo. Drug Deliv. 24 (1), 1360-1371. doi: 10.1080/10717544.2017.1375576

Wang, Y., Wang, S., Song, R., Cai, J., Xu, J., Tang, X., et al. (2019). Ginger polysaccharides induced cell cycle arrest and apoptosis in human hepatocellular carcinoma HepG2 cells. Int. J. Biol. Macromol. 123, 81-90. doi: 10.1016/j.ijbiomac.2018.10.169

Ward, C., Walker, A., Dransfield, I., Haslett, C., and Rossi, A. G. (2004). Regulation of granulocyte apoptosis by NF-kappaB. Biochem. Soc Trans. 32, 465-467. doi: 10.1042/bst0320465

Wei, Y., Qin, L., and Zhang, H. (2011). Cell apoptosis and signal transduction pathway. Int. J. Geriatr. 3 (2), 70-74. doi: 10.3969/j.issn.1674-7593.201102.006

Westhoff, M. A., and Fulda, S. (2009). Adhesion-mediated apoptosis resistance in cancer. Drug Resist. Updat. 12, 127-136. doi: 10.1016/j.drup.2009.08.001

Wu, Z., Sun, H., Li, J., Ma, C., Zhao, S., Guo, Z., et al. (2014). A polysaccharide from Sanguisorbae radix induces caspase-dependent apoptosis in human leukemia HL-60 cells. Int. J. Biol. Macromol. 70, 615-620. doi: 10.1016/ j.ijbiomac.2014.06.062

Wu, C., Luo, H., Ma, W., Ren, X., Lu, C., Li, N., et al. (2017). Polysaccharides isolated from Hedyotis diffusa inhibits the aggressive phenotypes of laryngeal squamous carcinoma cells via inhibition of Bcl-2, MMP-2, and muPA. Gene 637, 124-129. doi: 10.1016/j.gene.2017.09.041

Wu, Y. Z., Sun, J., and Wang, Y. B. (2017). Selective estrogen receptor modulator: A novel polysaccharide from Sparganii Rhizoma induces apoptosis in breast cancer cells. Carbohydr. Polym. 163, 199-207. doi: 10.1016/j.carbpol.2017.01.062

Wu, J., Wang, J., Su, Q., Ding, W., Li, T., Yu, J., et al. (2018a). Traditional Chinese medicine Astragalus polysaccharide enhanced antitumor effects of the angiogenesis inhibitor apatinib in pancreatic cancer cells on proliferation, invasiveness, and apoptosis. Oncol. Targets Ther. 11, 2685-2698. doi: 10.2147/ OTT.S157129

Wu, J., Yu, J., Wang, J., Zhang, C., Shang, K., Yao, X., et al. (2018b). Astragalus polysaccharide enhanced antitumor effects of Apatinib in gastric cancer AGS cells by inhibiting AKT signalling pathway. BioMed. Pharmacother. 100, 176183. doi: $10.1016 /$ j.biopha.2018.01.140

Xie, X. C., Zhao, N., Xu, Q. H., Yang, X., Xia, W. K., Chen, Q., et al. (2017). Relaxin attenuates aristolochic acid induced human tubular epithelial cell apoptosis in vitro by activation of the PI3K/Akt signaling pathway. Apoptosis 22 (6), 769776. doi: 10.1007/s10495-017-1369-z

Xie, P., Horio, F., Fujii, I., Zhao, J., Shinohara, M., and Matsukura, M. (2018). A novel polysaccharide derived from algae extract inhibits cancer progression via JNK, not via the p38 MAPK signaling pathway. Int. J. Oncol. 52 (5), 1380-1390. doi: $10.3892 /$ ijo.2018.4297

Xie, R.-D., Sun, S.-B., He, J.-X., Dong, J.-N., and Long, F. (2019). Effect of Astragalus Polysaccharide on Growth and Tumor-related Apoptosis Protein of Human Breast Cancer MDA-MB-231 Transplanted Tumor in Nude Mice. Chin. J. Exp. Tradit. Med. Formul. 25 (16), 37-43. doi: 10.13422/ j.cnki.syfjx.20191622

Xie, S. Z., Liu, B., Ye, H. Y., Li, Q. M., Pan, L. H., Zha, X. Q., et al. (2019). Dendrobium huoshanense polysaccharide regionally regulates intestinal mucosal barrier function and intestinal microbiota in mice. Carbohydr. Polym. 206, 149-162. doi: 10.1016/j.carbpol.2018.11.002

Xu, A. H., Chen, H. S., Sun, B. C., Xiang, X. R., Chu, Y. F., Zhai, F., et al. (2003). Therapeutic mechanism of ginkgo biloba exocarp polysaccharides on gastric cancer. World J. Gastroenterol. 9 (11), 2424-2427. doi: 10.3748/wjg.v9.i11.2424

$\mathrm{Xu}$, J. (2015). Effect of ginseng polysaccharide injection combined with chemotherapy on immune function and therapeutic of the patients with advanced gastric cancer. Zhongnan Pharma. 13 (3), 316-318,321. doi: 10.7539/j.issn.1672-2981.2015.03.025

Yaacoub, K., Pedeux, R., Tarte, K., and Guillaudeux, T. (2016). Role of the tumor microenvironment in regulating apoptosis and cancer progression. Cancer Lett. 378 (2), 150-159. doi: 10.1016/j.canlet.2016.05.012

Yamada, K., and Yoshida, K. (2019). Mechanical insights into the regulation of programmed cell death by $\mathrm{p} 53$ via mitochondria. Biochim. Biophys. Acta Mol. Cell Res. 1866 (5), 839-848. doi: 10.1016/j.bbamcr.2019.02.009

Yan, L.-j., Hong, T., Luo, J.-H., Cao, X.-M., Liu, W., Gao, Y., et al. (2017). Effect of Astragali Radix Polysaccharides on Proliferation and Apoptosis of Human Colon Cancer Cell Line SW620. Chin. J. Exp. Tradit. Med. Formul. 23 (22), 97101. doi: 10.13422/j.cnki.syfjx.2017220097

Yang, J., Shao, X., Jiang, J., Sun, Y., Wang, L., and Sun, L. (2018). Angelica sinensis polysaccharide inhibits proliferation, migration, and invasion by downregulating microRNA-675 in human neuroblastoma cell line SH-SY5Y. Cell Biol. Int. 42 (7), 867-876. doi: 10.1002/cbin.10954

Yang, K., Gao, Z. Y., Li, T. Q., Song, W., Xiao, W., Zheng, J., et al. (2019). Antitumor activity and the mechanism of a green tea (Camellia sinensis) polysaccharide on prostate cancer. Int. J. Biol. Macromol. 122, 95-103. doi: 10.1016/j.ijbiomac.2018.10.101

Yao, C., Cao, X., Fu, Z., Tian, J., Dong, W., Xu, J., et al. (2017). Boschniakia Rossica Polysaccharide Triggers Laryngeal Carcinoma Cell Apoptosis by Regulating Expression of Bcl-2, Caspase-3, and P53. Med. Sci. Monit. 23, 2059-2064. doi: $10.12659 / \mathrm{msm} .901381$

Yi, Y., Wang, H. X., and He, J. R. (2014). Research progresses of pharmacokinetics of polysaccharides. Acta Pharm. Sin. 49 (4), 443-449. doi: 10.16438/j.05134870.2014.04.012

Yokota, T., and Wang, Y. (2016). p38 MAP kinases in the heart. Gene 575, $369-$ 376. doi: 10.1016/j.gene.2015.09.030

Yu, L., Li, L., Medeiros, L. J., and Young, K. H. (2017). NF-кB signaling pathway and its potential as a target for therapy in lymphoid neoplasms. Blood Rev. 31 (2), 77-92. doi: 10.1016/j.blre.2016.10.001 
Yu, J., Ji, H., Dong, X., Feng, Y., and Liu, A. (2019). Apoptosis of human gastric carcinoma MGC-803 cells induced by a novel Astragalus membranaceus polysaccharide via intrinsic mitochondrial pathways. Int. J. Biol. Macromol. 126, 811-819. doi: 10.1016/j.ijbiomac.2018.12.268

Yuan, C., Wang, C., Wang, J., Kumar, V., Anwar, F., Xiao, F., et al. (2016). Inhibition on the growth of human MDA-MB-231 breast cancer cells in vitro and tumor growth in a mouse xenograft model by Se-containing polysaccharides from Pyracantha fortuneana. Nutr. Res. 36 (11), 1243-1254. doi: 10.1016/j.nutres.2016.09.012

Zabaiou, N., Fouache, A., Trousson, A., Buñay-Noboa, J., Marceau, G., Sapin, V., et al. (2019). Ethanolic extract of Algerian propolis decreases androgen receptor transcriptional activity in cultured LNCaP cells. J. Steroid Biochem. Mol. Biol. 189, 108-115. doi: 10.1016/j.jsbmb.2019.02.016

Zarei, O., Benvenuti, S., Ustun-Alkan, F., Hamzeh-Mivehroud, M., and Dastmalchi, S. (2016). Strategies of targeting the extracellular domain of RON tyrosine kinase receptor for cancer therapy and drug delivery. $J$. Cancer Res. Clin. Oncol. 142 (12), 2429-2446. doi: 10.1007/s00432-016-2214-4

Zeng, J., Dai, P., Ren, L., Song, B., Chen, X., Wang, X., et al. (2012). Apoptosisinduced anti-tumor effect of Curcuma kwangsiensis polysaccharides against human nasopharyngeal carcinoma cells. Carbohydr. Polym. 89 (4), 1067-1072. doi: 10.1016/j.carbpol.2012.03.064

Zeng, G., Shen, H., Tang, G., Cai, X., Bi, L., Sun, B., et al. (2015). A polysaccharide from the alkaline extract of Glycyrrhiza inflata induces apoptosis of human oral cancer SCC-25 cells via mitochondrial pathway. Tumour Biol. 36 (9), 6781-6788. doi: 10.1007/s13277-015-3359-5

Zhang, F., Song, X., Li, L., Wang, J., Lin, L., Li, C., et al. (2015). Polygala tenuifolia polysaccharide PTP induced apoptosis in ovarian cancer cells via a mitochondrial pathway. Tumour Biol. 36 (4), 2913-2919. doi: 10.1007/ s13277-014-2921-x

Zhang, F., Shi, J. J., Thakur, K., Hu, F., Zhang, J. G., and Wei, Z. J. (2017). AntiCancerous Potential of Polysaccharide Fractions Extracted from Peony Seed Dreg on Various Human Cancer Cell Lines Via Cell Cycle Arrest and Apoptosis. Front. Pharmacol. 8, 102. doi: 10.3389/fphar.2017.00102

Zhang, X. Y., Sun, K., Zhu, Q., Song, T., and Liu, Y. (2017). Ginseng polysaccharide serves as a potential radiosensitizer through inducing apoptosis and autophagy in the treatment of osteosarcoma. Kaohsiung J. Med. Sci. 33 (11), 535-542. doi: 10.1016/j.kjms.2017.07.001

Zhang, H. W., Lin, Z. X., Cheung, F., Cho, W. C., and Tang, J. L. (2018). Moxibustion for alleviating side effects of chemotherapy or radiotherapy in people with cancer. Cochrane Database Syst. Rev. 11, CD010559. doi: 10.1002/ 14651858.CD010559.pub2

Zhang, Q. Y., Wang, F. X., Jia, K. K., and Kong, L. D. (2018). Natural Product Interventions for Chemotherapy and Radiotherapy-Induced Side Effects. Front. Pharmacol. 9, 1253. doi: 10.3389/fphar.2018.01253

Zhang, W., He, W., Shi, X., Li, X., Wang, Y., Hu, M., et al. (2018). An Asparagus polysaccharide fraction inhibits MDSCs by inducing apoptosis through tolllike receptor 4. Phytother. Res. 32 (7), 1297-1303. doi: 10.1002/ptr.6058

Zhang, X., Zhao, S., Song, X., Jia, J., Zhang, Z., Zhou, H., et al. (2018). Inhibition effect of glycyrrhiza polysaccharide (GCP) on tumor growth through regulation of the gut microbiota composition. J. Pharmacol. Sci. 137 (4), 324-332. doi: 10.1016/j.jphs.2018.03.006

Zhang, K., Han, Y., Zhao, Y., Sun, Y., Zou, M., Fu, Y., et al. (2019). MGUpregulated gga-miR-16-5p Inhibits the Proliferation Cycle and Promotes the Apoptosis of -Infected DF-1 Cells by Repressing PIK3R1Mediated the PI3K/Akt/NF-KB Pathway to Exert Anti-Inflammatory Effect. Int. J. Mol. Sci. 20 (5), 1036-1052. doi: 10.3390/ijms20051036
Zhang, Q., Liu, J., Zhang, M., Wei, S., Li, R., Gao, Y., et al. (2019). Apoptosis Induction of Fibroblast-Like Synoviocytes Is an Important Molecular-Mechanism for Herbal Medicine along with its Active Components in Treating Rheumatoid Arthritis. Biomolecules 9 (12), 795-824. doi: 10.3390/biom9120795

Zhang, S. D., Yu, L., Wang, P., Kou, P., Li, J., Wang, L. T., et al. (2019). Inotodiol inhibits cells migration and invasion and induces apoptosis via p53-dependent pathway in HeLa cells. Phytomedicine 60, 152957. doi: 10.1016/ j.phymed.2019.152957

Zhao, J., Lu, Y., and Shen, H. M. (2012). Targeting p53 as a therapeutic strategy in sensitizing TRAIL-induced apoptosis in cancer cells. Cancer Lett. 314 (1), 8-23. doi: 10.1016/j.canlet.2011.09.040

Zhao, R., Gao, X., Cai, Y., Shao, X., Jia, G., Huang, Y., et al. (2013). Antitumor activity of Portulaca oleracea L. polysaccharides against cervical carcinoma in vitro and in vivo. Carbohydr. Polym. 96 (2), 376-383. doi: 10.1016/j.carbpol.2013.04.023

Zhao, R., Zhang, T., Ma, B., and Li, X. (2017). Antitumor Activity of Portulaca Oleracea L. Polysaccharide on HeLa Cells Through Inducing TLR4/NFkappaB Signaling. Nutr. Cancer 69 (1), 131-139. doi: 10.1080/ 01635581.2017.1248294

Zhao, Y., Sun, H., Ma, L., and Liu, A. (2017). Polysaccharides from the peels of Citrus aurantifolia induce apoptosis in transplanted $\mathrm{H} 22$ cells in mice. Int. J. Biol. Macromol. 101, 680-689. doi: 10.1016/j.ijbiomac.2017.03.149

Zhou, W. J., Wang, S., Hu, Z., Zhou, Z. Y., and Song, C. J. (2015). Angelica sinensis polysaccharides promotes apoptosis in human breast cancer cells via CREBregulated caspase-3 activation. Biochem. Biophys. Res. Commun. 467 (3), 562 569. doi: 10.1016/j.bbrc.2015.09.145

Zhou, X., Jiang, W., Liu, Z., Liu, S., and Liang, X. (2017). Virus Infection and Death Receptor-Mediated Apoptosis. Viruses 9 (11), 316-335. doi: 10.3390/v9110316

Zhou, Z., Meng, M., and Ni, H. (2017). Chemosensitizing Effect of Astragalus Polysaccharides on Nasopharyngeal Carcinoma Cells by Inducing Apoptosis and Modulating Expression of Bax/Bcl-2 Ratio and Caspases. Med. Sci. Monit. 23, 462-469. doi: $10.12659 / \mathrm{msm} .903170$

Zhou, H., Yang, J., Zhang, C., Zhang, Y., Wang, R., Li, X., et al. (2018). Safflower polysaccharide inhibits the development of tongue squamous cell carcinoma. World J. Surg. Oncol. 16 (1), 167. doi: 10.1186/s12957-018-1441-3

Zhu, Q., Chen, J., Li, Q., Wang, T., and Li, H. (2016). Antitumor activity of polysaccharide from Laminaria japonica on mice bearing $\mathrm{H} 22$ liver cancer. Int. J. Biol. Macromol. 92, 156-158. doi: 10.1016/j.ijbiomac.2016.06.090

Zong, A., Cao, H., and Wang, F. (2012). Anticancer polysaccharides from natural resources: a review of recent research. Carbohydr. Polym. 90 (4), 1395-1410. doi: 10.1016/j.carbpol.2012.07.026

Zou, Y., Xiong, H., Xiong, H., Lu, T., Zhu, F., Luo, Z., et al. (2015). A polysaccharide from Andrographis paniculata induces mitochondrialmediated apoptosis in human hepatoma cell line (HepG2). Tumour Biol. 36 (7), 5179-5186. doi: 10.1007/s13277-015-3172-1

Conflict of Interest: The authors declare that the research was conducted in the absence of any commercial or financial relationships that could be construed as a potential conflict of interest.

Copyright $\odot 2020$ Gan, Wang, Hu, Lou, Xiong, Peng and Huang. This is an openaccess article distributed under the terms of the Creative Commons Attribution License (CC BY). The use, distribution or reproduction in other forums is permitted, provided the original author(s) and the copyright owner(s) are credited and that the original publication in this journal is cited, in accordance with accepted academic practice. No use, distribution or reproduction is permitted which does not comply with these terms. 